\title{
A UNIFORM BOUND ON COSTS OF CONTROLLING SEMILINEAR HEAT EQUATIONS ON A SEQUENCE OF INCREASING DOMAINS AND ITS APPLICATION
}

\author{
LiJUAN WANG ${ }^{1,2}$ AND CAN ZHANG ${ }^{1,2, *}$
}

\begin{abstract}
In this paper, we first prove a uniform upper bound on costs of null controls for semilinear heat equations with globally Lipschitz nonlinearity on a sequence of increasing domains, where the controls are acted on an equidistributed set that spreads out in the whole Euclidean space $\mathbb{R}^{N}$. As an application, we then show the exact null-controllability for this semilinear heat equation in $\mathbb{R}^{N}$. The main novelty here is that the upper bound on costs of null controls for such kind of equations in large but bounded domains can be made uniformly with respect to the sizes of domains under consideration. The latter is crucial when one uses a suitable approximation argument to derive the global null-controllability for the semilinear heat equation in $\mathbb{R}^{N}$. This allows us to overcome the wellknown problem of the lack of compactness embedding arising in the study of null-controllability for nonlinear PDEs in generally unbounded domains.
\end{abstract}

Mathematics Subject Classification. 35K05, 93B07, 93C20.

Received May 11, 2021. Accepted January 4, 2022.

\section{INTRODUCTION AND MAIN RESULTS}

This paper is concerned with the null control costs for semilinear heat equations on a sequence of increasing bounded domains in $\mathbb{R}^{N}$ (with $N \in \mathbb{N}$ ), when the controls act on the interior subsets of these domains. Generally speaking, the null control costs depend on the geometry of both control regions and whole domains where the equations evolve. Nevertheless, the goal of this paper is to investigate the uniform upper estimate for null control costs with respect to the varying domains. As an interesting application, we shall derive the null-controllability of the semilinear heat equation in $\mathbb{R}^{N}$.

The general formulation of the problem could be stated as follows. Let $T$ be a positive time and let $E$ be a subset of positive Lebesgue measure in $(0, T)$. For each $n \in \mathbb{N}$, let $\omega_{n}$ be a nonempty open subset of bounded Lipschitz domain $\Omega_{n}$ in $\mathbb{R}^{N}$. Consider the following semilinear heat equation:

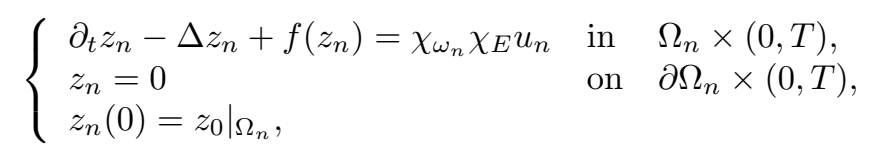

Keywords and phrases: Semilinear heat equation, null-controllability, uniform cost, equidistributed set.

1 School of Mathematics and Statistics, Wuhan University, Wuhan 430072, China.

2 Computational Science Hubei Key Laboratory, Wuhan University, Wuhan 430072, China.

* Corresponding author: zhangcansx@163.com 
where $z_{0} \in L^{2}\left(\mathbb{R}^{N}\right), u_{n}=u_{n}(x, t) \in L^{2}\left(\Omega_{n} \times(0, T)\right)$ is the control function, $\chi_{E}$ and $\chi_{\omega_{n}}$ are characteristic functions of $E$ and control region $\omega_{n}$, respectively. Throughout the paper, we suppose that $f$ is a globally Lipschitz function. Then, there exists a unique solution $z_{n} \in C\left([0, T] ; L^{2}\left(\Omega_{n}\right)\right) \bigcap L^{2}\left(0, T ; H_{0}^{1}\left(\Omega_{n}\right)\right)$ for the equation (1.1) (c.f., [11], for instance).

Recall that for each $n \in \mathbb{N}$, the control system (1.1) is called exactly null controllable at the time $T>0$, if the following statement is true: For any $z_{0} \in L^{2}\left(\mathbb{R}^{N}\right)$, there is a control $u_{n} \in L^{2}\left(0, T ; L^{2}\left(\Omega_{n}\right)\right)$ so that the corresponding solution $z_{n}$ to (1.1) satisfies that $z_{n}(T)=0$ in $\Omega_{n}$. Furthermore, the null control cost is the least constant $C_{n}$ so that $\left\|u_{n}\right\|_{L^{2}\left(\Omega_{n} \times(0, T)\right)} \leq C_{n}\left\|z_{0}\right\|_{L^{2}\left(\mathbb{R}^{N}\right)}$ holds for all $z_{0} \in L^{2}\left(\mathbb{R}^{N}\right)$. As mentioned at the beginning, the constant $C_{n}$ may depend on the geometry parameters of $\Omega_{n}$ and $\omega_{n}$.

The question whether or not a given control system is null controllable and obtaining upper bounds for the associated null control costs, with respect to the time interval or control regions, are important topics in control theory, both for linear partial differential control equations and abstract linear control systems. We refer the reader to $[1,3,9,10,17,18,25,26,28]$ and references therein for a wider discussion on this activated research field.

Meanwhile, there are many fascinating works in the literature on the approximate or exact null-controllability for the semilinear heat equations in bounded domains; see, for instance, [11, 13, 21]. Their proofs are usually divided into two parts: (i) null-controllability of the linearized system; (ii) a fixed-point argument. The global null-controllability was also proved for a class of nonlinearities for which blow-up phenomena may arise (see, e.g., $[2,12,15])$.

To the best of our knowledge, there are very few works on establishing a uniform upper bound for the null control cost for a partial differential controlled equation in varying domains.

In this paper, we shall restrict the control on an equidistributed set. We say a set as an equidistributed set in $\mathbb{R}^{N}$ if it contains a union of suitably distributed balls of fixed radius. Recently, there are many beautiful existing results on the quantitative unique continuation for general elliptic operators on equidistributed sets (c.f., $[10,17,23]$ and references therein). Meanwhile, we will consider the varying domains $\Omega_{n}$ as an approximation of $\mathbb{R}^{N}$, for the simplicity.

In order to state our main results, we first introduce certain standard notations. For each $r>0$ and $x_{0} \in \mathbb{R}^{N}$, $B_{r}\left(x_{0}\right)$ stands for the closed ball centered at $x_{0}$ and of radius $r ; Q_{r}\left(x_{0}\right)$ denotes the smallest closed cube centered at $x_{0}$ so that $B_{r}\left(x_{0}\right) \subset Q_{r}\left(x_{0}\right)$; $\operatorname{int}\left(Q_{r}\left(x_{0}\right)\right)$ is the interior of $Q_{r}\left(x_{0}\right)$.

Let $0<r_{1}<r_{2}<\infty$. The following three assumptions will be effective throughout the paper:

$\left(H_{1}\right)$. There is a sequence $\left\{x_{i}\right\}_{i=1}^{\infty} \subset \mathbb{R}^{N}$ so that $\mathbb{R}^{N}=\bigcup_{i=1}^{\infty} Q_{r_{2}}\left(x_{i}\right), \operatorname{int}\left(Q_{r_{2}}\left(x_{i}\right)\right) \bigcap \operatorname{int}\left(Q_{r_{2}}\left(x_{j}\right)\right)=\emptyset$ for each $i \neq j$. Moreover, $\omega \triangleq \bigcup_{i=1}^{\infty} \omega_{i}$, where $\omega_{i}$ is an open set and $B_{r_{1}}\left(x_{i}\right) \subset \omega_{i} \subset B_{r_{2}}\left(x_{i}\right)$ for each $i \in \mathbb{N}$.

$\left(H_{2}\right)$. For each $n \in \mathbb{N}, \Omega_{n} \triangleq \operatorname{int}\left(\bigcup_{i \in I_{n}} Q_{r_{2}}\left(x_{i}\right)\right)$ is convex, with Card $\left(I_{n}\right)<\infty$. In addition, $I_{n} \subsetneq I_{m}$ when $n<m$, and $\bigcup_{n=1}^{\infty} \Omega_{n}=\mathbb{R}^{N}$.

$\left(H_{3}\right)$. The function $f: \mathbb{R} \rightarrow \mathbb{R}$ is globally Lipschitz, i.e., $|f(s)-f(\tau)| \leq L|s-\tau|$ for all $s, \tau \in \mathbb{R}$ with some constant $L>0$, and satisfies that $f(0)=0$.

With regarding to the assumption $\left(H_{1}\right)$, we may say that the set $\omega$ is an equidistributed set in $\mathbb{R}^{N}$. A particularly example is a periodic arrangement of balls (see Fig. 1). Very recently, we have proved in [8] the observability inequality and null-controllability on such kind of sets for the linear heat equation with time and space dependent potentials in $\mathbb{R}^{N}$. It is worthing mentioning that the authors in [16] imposed a sufficient condition on the observation subdomain so that the corresponding observability inequality holds for the Kolmogorov equation in the whole domain. 


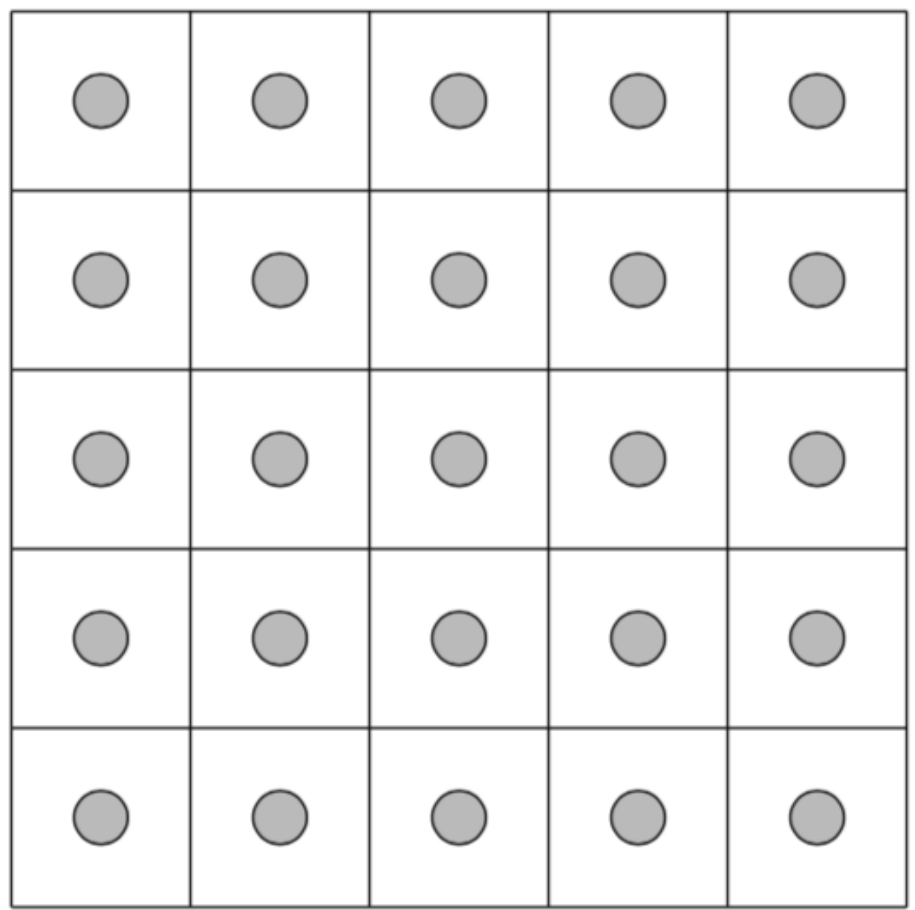

Figure 1. Example.

The first main result of this paper concerning the uniform upper bound on costs of controlling a semilinear heat equation on increasing large domains can be stated as follows.

Theorem 1.1. Let $\left(H_{1}\right),\left(H_{2}\right)$ and $\left(H_{3}\right)$ hold. Let $T>0$ and $E$ be a subset of positive measure in $(0, T)$. Then there is a positive constant $\kappa=\kappa\left(r_{1}, r_{2}, E, T, L\right)$ so that for any $n \in \mathbb{N}$ and any $z_{0} \in L^{2}\left(\mathbb{R}^{N}\right)$, there is a control $u_{n} \in L^{2}\left(\mathbb{R}^{N} \times(0, T)\right)$, with the uniform bound

$$
\left\|u_{n}\right\|_{L^{2}\left(\mathbb{R}^{N} \times(0, T)\right)} \leq \kappa\left\|z_{0}\right\|_{L^{2}\left(\mathbb{R}^{N}\right)}
$$

so that the corresponding solution $z_{n} \in C\left([0, T] ; L^{2}\left(\Omega_{n}\right)\right)$ to the following semilinear heat equation

$$
\begin{cases}\partial_{t} z_{n}-\Delta z_{n}+f\left(z_{n}\right)=\chi_{\omega \cap \Omega_{n}} \chi_{E} u_{n} & \text { in } \Omega_{n} \times(0, T), \\ z_{n}=0 & \text { on } \partial \Omega_{n} \times(0, T), \\ z_{n}(0)=z_{0} & \text { in } \Omega_{n},\end{cases}
$$

satisfies that $z_{n}(T)=0$ over $\Omega_{n}$.

Remark 1.2. Note that the control here is allowed to be activated on measurable sets of positive Lebesgue measure in the time variable rather than on the whole interval. Such a kind of refined null-controllability is useful in deriving the bang-bang property for the time optimal control problem of semilinear heat equations (see, for instance, [21]).

Remark 1.3. Our argument does not allow us to establish a similar result for the global null-controllability of slightly superlinearities as studied in $[2,12,15]$. 
Remark 1.4. We do not know how to extend this result from an equidistributed set $\omega$ to a more general thick set. We refer the reader to Remark 1.7 of [8] for the difficulty.

Another motivation of this paper is to establish the null-controllability for the semilinear heat equation in the whole space $\mathbb{R}^{N}$, when the control is acted on an equidistributed set. As already remarked in Remark 3.5 of our recent work [8] on the null-controllability for the linear heat equation with bounded potentials, the linearized plus fixed-point approach in $[11,13]$ cannot be directly applied in the framework of general unbounded domains because of the lack of compactness of Sobolev's embedding.

The authors of [7] studied the approximate controllability of a semilinear heat equation in an unbounded domain $\mathcal{O}$ of $\mathbb{R}^{N}$, with control only acted in an open and nonempty subset, by an approximation method. More precisely, they first considered the approximate controllability problem in bounded domains of the form $\mathcal{O}_{n} \triangleq \mathcal{O} \bigcap B_{n}$, where $B_{n}$ denotes the ball centered at the origin and of radius $n$; and then they showed that the controls proposed in [11] restricted to $\mathcal{O}_{n}$ converge in some sense to a desired approximate control in $\mathcal{O}$, as $n$ goes to infinity. One of main ingredients in their proofs is a qualitative unique continuation property for the linear parabolic equation. Nevertheless, the technical proof of [7] (see also [6]) is not valid any more for the null-controllability of a semilinear heat equation in $\mathbb{R}^{N}$.

It is also mentioning that the authors in $[4,14]$ proved the null-controllability for some semilinear heat equations in an unbounded domain $\mathcal{O}$ of $\mathbb{R}^{N}$, when the control is assumed to be distributed along a subdomain $\omega$ so that the uncontrolled region $\mathcal{O} \backslash \omega$ is bounded. The arguments therein are mainly based on a Carleman estimate for the linear parabolic operator in bounded domains.

Recently, the authors in [23] (see also [10]) showed in a linear and abstract framework that if the sequence of null controls associated to an exhaustion of an unbounded domain converges, then the solutions converge in the same way to the limiting problem on the unbounded domain. This result allows to infer the null-controllability on unbounded domain by studying the control problem on a sequence of bounded domains. In particular, it recovers the null-controllability result for the linear heat equation in $\mathbb{R}^{N}$. The latter has been already established well in $[9,25]$ independently.

Inspired by these works, we could utilize Theorem 1.1 to prove the null-controllability for a semilinear heat equation in $\mathbb{R}^{N}$ with the control acted on an equidistributed set $\omega$. In fact, since null controls in Theorem 1.1 are uniformly bounded, $\left\{u_{n}\right\}_{n \geq 1}$ has a weakly convergent subsequence with a limit $u$ in $L^{2}\left(\mathbb{R}^{N} \times(0, T)\right)$. Thus, one may expect that such control maybe a null control for the corresponding semilinear heat controlled equation in $\mathbb{R}^{N}$. Actually we have the following result:

Theorem 1.5. Assume that $\left(H_{1}\right)$ and $\left(H_{3}\right)$ hold. Let $T>0$ and $E$ be a subset of positive measure in $(0, T)$. Then, for each initial value $y_{0} \in L^{2}\left(\mathbb{R}^{N}\right)$, there is a control $u \in L^{2}\left(0, T ; L^{2}\left(\mathbb{R}^{N}\right)\right)$ with an upper bound

$$
\|u\|_{L^{2}\left(\mathbb{R}^{N} \times(0, T)\right)} \leq \kappa\left\|y_{0}\right\|_{L^{2}\left(\mathbb{R}^{N}\right)},
$$

so that the corresponding solution

$$
\left\{\begin{array}{lll}
\partial_{t} y-\Delta y+f(y)=\chi_{\omega} \chi_{E} u & \text { in } \mathbb{R}^{N} \times(0, T) \\
y(0)=y_{0} & \text { in } \mathbb{R}^{N}
\end{array}\right.
$$

satisfies that $y(T)=0$ in $\mathbb{R}^{N}$. Here $\kappa$ is the same constant as in Theorem 1.1.

Remark 1.6. The well-posedness of such kind of semilinear heat equations in general unbounded domains is stated without a proof in [4], for instance. We refer the reader to Definition 4.1 and Lemma 4.2 in Section 4 for precise presentations.

Remark 1.7. Instead of $\mathbb{R}^{N}$, our considerations are valid as well for every set that can be approximated with cubes such as the half space $\mathbb{R}^{N-1} \times \mathbb{R}^{+}$and the infinite strip.

Remark 1.8. Our method does not rely on any compactness argument in the whole space $\mathbb{R}^{N}$. 
The rest of this paper is organized as follows. Section 2 is devoted to prove the uniform bound of control costs for the linearized controlled system. Section 3 shows the proof of Theorem 1.1 by a fixed-point argument. Finally, Section 4 presents the proof of Theorem 1.5.

\section{THE LINEAR CASE}

For each $n \in \mathbb{N}$, let $\varphi_{n}$ be the solution to the following linear heat equation with a bounded potential $a \in L^{\infty}\left(\mathbb{R}^{N} \times(0, T)\right)$ :

$$
\begin{cases}\partial_{t} \varphi_{n}-\Delta \varphi_{n}+a \varphi_{n}=0 & \text { in } \quad \Omega_{n} \times(0, T) \\ \varphi_{n}=0 & \text { on } \partial \Omega_{n} \times(0, T) \\ \varphi_{n}(0)=\varphi_{0} & \text { in } \quad \Omega_{n}\end{cases}
$$

with $\varphi_{0} \in L^{2}\left(\mathbb{R}^{N}\right)$.

We first state a uniform observability inequality for all solutions of $(2.1)$ evolving in $\Omega_{n}$ for all $n \in \mathbb{N}$. Here and throughout this paper, we simply write $\|a\|_{\infty}=\|a\|_{L^{\infty}\left(\mathbb{R}^{N} \times(0, T)\right)}$; and write $C(\cdot)$ for a positive constant depending on what are enclosed in the brackets.

Theorem 2.1. Assume that $\left(H_{1}\right)$ and $\left(H_{2}\right)$ hold. Let $T>0$ and $E$ be a subset of positive measure in $(0, T)$. Then there are constants $C=C\left(r_{1}, r_{2}\right)$ and $\widetilde{C}=\widetilde{C}\left(r_{1}, r_{2}, E\right)$ so that the following observability inequality

$$
\int_{\Omega_{n}}\left|\varphi_{n}(x, T)\right|^{2} \mathrm{~d} x \leq e^{\widetilde{C}} e^{C\left(T+T\|a\|_{\infty}+\|a\|_{\infty}^{2 / 3}\right)} \int_{E} \int_{\omega \cap \Omega_{n}}\left|\varphi_{n}(x, t)\right|^{2} \mathrm{~d} x \mathrm{~d} t, \quad \forall \varphi_{0} \in L^{2}\left(\mathbb{R}^{N}\right),
$$

holds uniformly for all $n \in \mathbb{N}$.

Remark 2.2. Note that the two constants in the above theorem are independent of the sizes of domains.

Remark 2.3. Under the same assumptions as in Theorem 2.1, the following refined estimate is also true: There are constants $C=C\left(r_{1}, r_{2}\right)$ and $\widetilde{C}=\widetilde{C}\left(r_{1}, r_{2}, E\right)$ so that the $L^{1}$-type observability inequality

$$
\left\|\varphi_{n}(T)\right\|_{L^{2}\left(\Omega_{n}\right)} \leq e^{\widetilde{C}} e^{C\left(T+T\|a\|_{\infty}+\|a\|_{\infty}^{2 / 3}\right)} \int_{E} \int_{\omega \cap \Omega_{n}}\left|\varphi_{n}(x, t)\right| \mathrm{d} x \mathrm{~d} t, \quad \forall \varphi_{0} \in L^{2}\left(\mathbb{R}^{N}\right),
$$

holds uniformly for all $n \in \mathbb{N}$.

As a direct consequence of Theorem 2.1, we present the linear version of Theorem 1.1 as follows. Consider the following linear control system:

$$
\begin{cases}\partial_{t} z_{n}-\Delta z_{n}+a z_{n}=\chi_{\omega} \cap \Omega_{n} \chi_{E} u_{n} & \text { in } \Omega_{n} \times(0, T), \\ z_{n}=0 & \text { on } \partial \Omega_{n} \times(0, T), \\ z_{n}(0)=z_{0} & \text { in } \Omega_{n},\end{cases}
$$

where $z_{0} \in L^{2}\left(\mathbb{R}^{N}\right)$ is an initial state and $u_{n} \in L^{2}\left(\Omega_{n} \times(0, T)\right)$ is a control. For each $n \in \mathbb{N}$, we write $z_{n}\left(\cdot ; z_{0}, u_{n}\right)$ for the solution to (2.2). By a standard duality method (see, for instance, [5], Thm. 2.42), we can easily obtain the following null-controllability result with a uniform upper bound on control costs.

Corollary 2.4. Under the same assumptions of Theorem 2.1, for each $n \in \mathbb{N}$ and $z_{0} \in L^{2}\left(\mathbb{R}^{N}\right)$, there is a control $u_{n} \in L^{2}\left(0, T ; L^{2}\left(\mathbb{R}^{N}\right)\right)$, with a uniform cost

$$
\left\|u_{n}\right\|_{L^{2}\left(0, T ; L^{2}\left(\mathbb{R}^{N}\right)\right)} \leq e^{\widetilde{C}} e^{C\left(T+T\|a\|_{\infty}+\|a\|_{\infty}^{2 / 3}\right)}\left\|z_{0}\right\|_{L^{2}\left(\mathbb{R}^{N}\right)},
$$


where the constants $C$ and $\widetilde{C}$ are given by Theorem 2.1, so that $z_{n}\left(T ; z_{0}, u_{n}\right)=0$ in $\Omega_{n}$.

\subsection{Quantitative estimates of unique continuation}

Before giving the proof of Theorem 2.1, we present the following quantitative unique continuation property for all solutions of (2.1).

Proposition 2.5. Let $\left(H_{2}\right)$ hold. Let $0<r<R<\infty$ and $\delta \in(0,1]$. Then there are a universal constant $C>0$ and three positive constants $C_{1} \triangleq C_{1}(R, \delta), C_{2} \triangleq C_{2}(R, \delta)$ and $\gamma \triangleq \gamma(r, R, \delta) \in(0,1)$ so that for any $n \in \mathbb{N}$, any $x_{0} \in \Omega_{n}$, and any $\varphi_{0} \in L^{2}\left(\Omega_{n}\right)$, the solution $\varphi_{n}$ of (2.1) satisfies

$$
\begin{aligned}
\int_{B_{R}\left(x_{0}\right) \cap \Omega_{n}}\left|\varphi_{n}(x, T)\right|^{2} \mathrm{~d} x \leq & {\left[C_{1} e^{\left[1+2 C\left(1+\frac{1}{R^{2}}\right)\right]\left(1+\frac{4}{T}+\|a\|_{\infty}^{2 / 3}\right)+\frac{C_{2}}{T}+2 T\|a\|_{\infty}} \int_{T / 2}^{T} \int_{Q_{2 R_{0}}\left(x_{0}\right) \cap \Omega_{n}} \varphi_{n}^{2}(x, t) \mathrm{d} x \mathrm{~d} t\right]^{\gamma} } \\
& \times\left(2 \int_{B_{r}\left(x_{0}\right) \cap \Omega_{n}}\left|\varphi_{n}(x, T)\right|^{2} \mathrm{~d} x\right)^{1-\gamma},
\end{aligned}
$$

where $R_{0} \triangleq(1+2 \delta) R$.

Remark 2.6. Note that the constants above are independent of the sizes of the domains $\Omega_{n}$.

The proof of Proposition 2.5 is very similar to the proof of Lemma 3.2 in [8]. For the sake of completeness, we provide its detailed proof in the appendix. Based on Proposition 2.5, we then could obtain a global interpolation inequality for solutions of (2.1) at one point of time variable.

Proposition 2.7. Assume that $\left(H_{1}\right)$ and $\left(H_{2}\right)$ hold. Then there are two constants $C_{3} \triangleq C_{3}\left(r_{2}\right)>0$ and $\theta \triangleq$ $\theta\left(r_{1}, r_{2}\right) \in(0,1)$ so that for any $n \in \mathbb{N}$ and any $\varphi_{0} \in L^{2}\left(\Omega_{n}\right)$, the solution $\varphi_{n}$ of (2.1) satisfies

$$
\int_{\Omega_{n}}\left|\varphi_{n}(x, T)\right|^{2} \mathrm{~d} x \leq e^{C_{3}\left(T^{-1}+T+T\|a\|_{\infty}+\|a\|_{\infty}^{2 / 3}\right)}\left(\int_{\Omega_{n}}\left|\varphi_{0}(x)\right|^{2} \mathrm{~d} x\right)^{\theta}\left(\int_{\omega \cap \Omega_{n}}\left|\varphi_{n}(x, T)\right|^{2} \mathrm{~d} x\right)^{1-\theta} .
$$

Proof. According to Proposition 2.5 (where $x_{0}, r, R$ and $\delta$ are replaced by $x_{i} \in \Omega_{n}, r_{1}, \sqrt{N} r_{2}$ and $1 / 2$, respectively) and the fact that $Q_{r_{2}}\left(x_{i}\right) \subset B_{\sqrt{N} r_{2}}\left(x_{i}\right)$, we obtain

$$
\begin{aligned}
& \int_{\operatorname{int}\left(Q_{r_{2}}\left(x_{i}\right)\right)}\left|\varphi_{n}(x, T)\right|^{2} \mathrm{~d} x \leq \int_{B_{\sqrt{N} r_{2}}\left(x_{i}\right) \cap \Omega_{n}}\left|\varphi_{n}(x, T)\right|^{2} \mathrm{~d} x \\
\leq & {\left[\widehat{\mathcal{K}}_{1} e^{\left[1+2 C\left(1+r_{2}^{-2}\right)\right]\left(1+4 T^{-1}+\|a\|_{\infty}^{2 / 3}\right)+\widehat{\mathcal{K}}_{2} T^{-1}+2 T\|a\|_{\infty}} \int_{T / 2}^{T} \int_{Q_{4 \sqrt{N} r_{2}}\left(x_{i}\right) \cap \Omega_{n}} \varphi_{n}^{2} \mathrm{~d} x \mathrm{~d} t\right]^{\theta} } \\
& \times\left[2 \int_{B_{r_{1}}\left(x_{i}\right) \cap \Omega_{n}}\left|\varphi_{n}(x, T)\right|^{2} \mathrm{~d} x\right]^{1-\theta},
\end{aligned}
$$

where $\widehat{\mathcal{K}}_{1} \triangleq \widehat{\mathcal{K}}_{1}\left(r_{2}\right)>0, \widehat{\mathcal{K}}_{2} \triangleq \widehat{\mathcal{K}}_{2}\left(r_{2}\right)>0$ and $\theta \triangleq \theta\left(r_{1}, r_{2}\right) \in(0,1)$. This, along with Young's inequality, implies that for each $\varepsilon>0$,

$$
\int_{\operatorname{int}\left(Q_{r_{2}}\left(x_{i}\right)\right)}\left|\varphi_{n}(x, T)\right|^{2} \mathrm{~d} x
$$




$$
\begin{aligned}
\leq & \varepsilon \theta \widehat{\mathcal{K}}_{1} e^{\left[1+2 C\left(1+r_{2}^{-2}\right)\right]\left(1+4 T^{-1}+\|a\|_{\infty}^{2 / 3}\right)+\widehat{\mathcal{K}}_{2} T^{-1}+2 T\|a\|_{\infty}} \int_{T / 2}^{T} \int_{Q_{4 \sqrt{N} r_{2}}\left(x_{i}\right) \cap \Omega_{n}} \varphi_{n}^{2} \mathrm{~d} x \mathrm{~d} t \\
& +2 \varepsilon^{-\frac{\theta}{1-\theta}}(1-\theta) \int_{B_{r_{1}}\left(x_{i}\right) \cap \Omega_{n}}\left|\varphi_{n}(x, T)\right|^{2} \mathrm{~d} x .
\end{aligned}
$$

By $\left(H_{2}\right)$, we have

$$
\begin{aligned}
& \int_{\Omega_{n}}\left|\varphi_{n}(x, T)\right|^{2} \mathrm{~d} x=\sum_{i \in I_{n}} \int_{\operatorname{int}\left(Q_{r_{2}}\left(x_{i}\right)\right)}\left|\varphi_{n}(x, T)\right|^{2} \mathrm{~d} x \\
\leq & \varepsilon \theta \widehat{\mathcal{K}}_{1} e^{\left[1+2 C\left(1+r_{2}^{-2}\right)\right]\left(1+4 T^{-1}+\|a\|_{\infty}^{2 / 3}\right)+\widehat{\mathcal{K}}_{2} T^{-1}+2 T\|a\|_{\infty}} \sum_{i \in I_{n}} \int_{T / 2}^{T} \int_{Q_{4 \sqrt{N} r_{2}}\left(x_{i}\right) \cap \Omega_{n}} \varphi_{n}^{2} \mathrm{~d} x \mathrm{~d} t \\
& +2 \varepsilon^{-\frac{\theta}{1-\theta}}(1-\theta) \int_{\omega \cap \Omega_{n}}\left|\varphi_{n}(x, T)\right|^{2} \mathrm{~d} x .
\end{aligned}
$$

Denote by

$$
\widetilde{\varphi}_{n}(x, t) \triangleq\left\{\begin{array}{lll}
\varphi_{n}(x, t) & \text { if } \quad(x, t) \in \Omega_{n} \times(0, T) \\
0 & \text { if } \quad(x, t) \in\left(\mathbb{R}^{N} \backslash \Omega_{n}\right) \times(0, T) .
\end{array}\right.
$$

We can directly check that

$$
\begin{aligned}
& \sum_{i \in I_{n}} \int_{T / 2}^{T} \int_{Q_{4 \sqrt{N} r_{2}}\left(x_{i}\right) \cap \Omega_{n}} \varphi_{n}^{2} \mathrm{~d} x \mathrm{~d} t \\
\leq & \sum_{i \geq 1} \int_{T / 2}^{T} \int_{Q_{4 \sqrt{N} r_{2}}\left(x_{i}\right)}\left(\chi_{\Omega_{n}} \widetilde{\varphi}_{n}\right)^{2} \mathrm{~d} x \mathrm{~d} t \\
\leq & \widehat{\mathcal{K}}_{3} \int_{T / 2}^{T} \int_{\mathbb{R}^{N}}\left(\chi_{\Omega_{n}} \widetilde{\varphi}_{n}\right)^{2} \mathrm{~d} x \mathrm{~d} t=\widehat{\mathcal{K}}_{3} \int_{T / 2}^{T} \int_{\Omega_{n}} \varphi_{n}^{2} \mathrm{~d} x \mathrm{~d} t,
\end{aligned}
$$

where $\widehat{\mathcal{K}}_{3}>0$. Then it follows from (2.4) that

$$
\begin{aligned}
& \int_{\Omega_{n}}\left|\varphi_{n}(x, T)\right|^{2} \mathrm{~d} x \\
\leq & \varepsilon \theta \widehat{\mathcal{K}}_{1} \widehat{\mathcal{K}}_{3} e^{\left[1+2 C\left(1+r_{2}^{-2}\right)\right]\left(1+4 T^{-1}+\|a\|_{\infty}^{2 / 3}\right)+\widehat{\mathcal{K}}_{2} T^{-1}+2 T\|a\|_{\infty}} \int_{T / 2}^{T} \int_{\Omega_{n}} \varphi_{n}^{2} \mathrm{~d} x \mathrm{~d} t \\
& +2 \varepsilon^{-\frac{\theta}{1-\theta}}(1-\theta) \int_{\omega \cap \Omega_{n}}\left|\varphi_{n}(x, T)\right|^{2} \mathrm{~d} x \text { for each } \varepsilon>0 .
\end{aligned}
$$

Notice that for any postive numbers $a_{1}, a_{2}, a_{3}$ and $\theta \in(0,1)$,

$$
\begin{aligned}
& a_{1} \leq a_{2}^{\theta} a_{3}^{1-\theta} \\
\Leftrightarrow & a_{1} \leq \varepsilon a_{2}+(1-\theta) \theta^{\frac{\theta}{1-\theta}} \varepsilon^{-\frac{\theta}{1-\theta}} a_{3} \text { for each } \varepsilon>0 .
\end{aligned}
$$


The above equivalence, along with (2.5), implies

$$
\begin{aligned}
& \int_{\Omega_{n}}\left|\varphi_{n}(x, T)\right|^{2} \mathrm{~d} x \\
\leq & {\left[\widehat{\mathcal{K}}_{1} \widehat{\mathcal{K}}_{3} e^{\left[1+2 C\left(1+r_{2}^{-2}\right)\right]\left(1+4 T^{-1}+\|a\|_{\infty}^{2 / 3}\right)+\widehat{\mathcal{K}}_{2} T^{-1}+2 T\|a\|_{\infty}} \int_{T / 2}^{T} \int_{\Omega_{n}} \varphi_{n}^{2} \mathrm{~d} x \mathrm{~d} t\right]^{\theta} } \\
& \times\left[2 \int_{\omega \cap \Omega_{n}}\left|\varphi_{n}(x, T)\right|^{2} \mathrm{~d} x\right]^{1-\theta} .
\end{aligned}
$$

Noting that

$$
\int_{\Omega_{n}}\left|\varphi_{n}(x, t)\right|^{2} \mathrm{~d} x \leq e^{2\|a\|_{\infty} t} \int_{\Omega_{n}}\left|\varphi_{0}(x)\right|^{2} \mathrm{~d} x \quad \text { for each } t \in[0, T]
$$

by (2.6), we deduce

$$
\begin{aligned}
\int_{\Omega_{n}}\left|\varphi_{n}(x, T)\right|^{2} \mathrm{~d} x \leq & {\left[\widehat{\mathcal{K}}_{1} \widehat{\mathcal{K}}_{3} T e^{\left[1+2 C\left(1+r_{2}^{-2}\right)\right]\left(1+4 T^{-1}+\|a\|_{\infty}^{2 / 3}\right)+\widehat{\mathcal{K}}_{2} T^{-1}+2 T\|a\|_{\infty}} e^{2 T\|a\|_{\infty}} \int_{\Omega_{n}} \varphi_{0}^{2} \mathrm{~d} x\right]^{\theta} } \\
& \times\left[2 \int_{\omega \cap \Omega_{n}}\left|\varphi_{n}(x, T)\right|^{2} \mathrm{~d} x\right]^{1-\theta}
\end{aligned}
$$

Hence, (2.3) follows from the latter inequality immediately.

\subsection{Proof of Theorem 2.1}

Now, we are able to present the proof of Theorem 2.1 by using the telescoping series method. The proof is similar to that of Theorem 1.1 in [8]. Here we only sketch the proof.

Proof of Theorem 2.1. Arbitrarily fix $n \in \mathbb{N}$. For any $0 \leq t_{1}<t_{2} \leq T$, by a translation in the time variable and Proposition 2.7, we obtain from Young's inequality that

$$
\left\|\varphi_{n}\left(t_{2}\right)\right\|_{L^{2}\left(\Omega_{n}\right)}^{2} \leq \varepsilon\left\|\varphi_{n}\left(t_{1}\right)\right\|_{L^{2}\left(\Omega_{n}\right)}^{2}+\frac{\widetilde{\mathcal{K}}_{1}}{\varepsilon^{\alpha}} e^{\frac{\tilde{\mathcal{K}}_{2}}{t_{2}-t_{1}}}\left\|\varphi_{n}\left(t_{2}\right)\right\|_{L^{2}\left(\omega \cap \Omega_{n}\right)}^{2} \quad \text { for each } \varepsilon>0
$$

where $\widetilde{\mathcal{K}}_{1} \triangleq e^{\frac{C_{3}}{1-\theta}\left(T+T\|a\|_{\infty}+\|a\|_{\infty}^{2 / 3}\right)}, \widetilde{\mathcal{K}}_{2} \triangleq C_{3} /(1-\theta)$ and $\alpha \triangleq \theta /(1-\theta)$.

Let $l$ be a density point of $E$, i.e., $l \in E$ and

$$
\lim _{\sigma \rightarrow 0} \frac{|(l-\sigma, l+\sigma) \bigcap E|}{2 \sigma}=1 .
$$

According to Proposition 2.1 in [20], for each $\kappa>1$, there exists $l_{1} \in(l, T)$, depending on $\kappa$ and $E$, so that the sequence $\left\{l_{m}\right\}_{m \geq 1}$, given by

$$
l_{m+1}=l+\frac{1}{\kappa^{m}}\left(l_{1}-l\right),
$$


satisfies

$$
l_{m}-l_{m+1} \leq 3\left|E \bigcap\left(l_{m+1}, l_{m}\right)\right|
$$

Next, let $0<l_{m+2}<l_{m+1} \leq t<l_{m}<l_{1}<T$. It follows from (2.7) that

$$
\left\|\varphi_{n}(t)\right\|_{L^{2}\left(\Omega_{n}\right)}^{2} \leq \varepsilon\left\|\varphi_{n}\left(l_{m+2}\right)\right\|_{L^{2}\left(\Omega_{n}\right)}^{2}+\frac{\widetilde{\mathcal{K}}_{1}}{\varepsilon^{\alpha}} e^{\frac{\widetilde{\mathcal{K}}_{2}}{t-l} m+2}\left\|\varphi_{n}(t)\right\|_{L^{2}\left(\omega \cap \Omega_{n}\right)}^{2} \text { for each } \varepsilon>0 .
$$

By a standard energy estimate, we have

$$
\left\|\varphi_{n}\left(l_{m}\right)\right\|_{L^{2}\left(\Omega_{n}\right)} \leq e^{T\|a\|_{\infty}}\left\|\varphi_{n}(t)\right\|_{L^{2}\left(\Omega_{n}\right)} .
$$

This, along with (2.9), implies

$$
\left\|\varphi_{n}\left(l_{m}\right)\right\|_{L^{2}\left(\Omega_{n}\right)}^{2} \leq e^{2 T\|a\|_{\infty}}\left(\varepsilon\left\|\varphi_{n}\left(l_{m+2}\right)\right\|_{L^{2}\left(\Omega_{n}\right)}^{2}+\frac{\widetilde{\mathcal{K}}_{1}}{\varepsilon^{\alpha}} e^{\frac{\widetilde{\mathcal{K}}_{2}}{t-l} m+2}\left\|\varphi_{n}(t)\right\|_{L^{2}\left(\omega \cap \Omega_{n}\right)}^{2}\right) \text { for each } \varepsilon>0
$$

which indicates that

$$
\left\|\varphi_{n}\left(l_{m}\right)\right\|_{L^{2}\left(\Omega_{n}\right)}^{2} \leq \varepsilon\left\|\varphi_{n}\left(l_{m+2}\right)\right\|_{L^{2}\left(\Omega_{n}\right)}^{2}+\frac{\widetilde{\mathcal{K}}_{3}}{\varepsilon^{\alpha}} e^{\frac{\widetilde{\mathcal{K}}_{2}}{t-l_{m+2}}}\left\|\varphi_{n}(t)\right\|_{L^{2}\left(\omega \cap \Omega_{n}\right)}^{2} \text { for each } \varepsilon>0,
$$

where $\widetilde{\mathcal{K}}_{3}=\left(e^{2 T\|a\|_{\infty}}\right)^{1+\alpha} \widetilde{\mathcal{K}}_{1}$. Integrating the latter inequality over $E \bigcap\left(l_{m+1}, l_{m}\right)$ gives

$$
\begin{aligned}
\left|E \bigcap\left(l_{m+1}, l_{m}\right)\right|\left\|\varphi_{n}\left(l_{m}\right)\right\|_{L^{2}\left(\Omega_{n}\right)}^{2} \leq & \varepsilon\left|E \bigcap\left(l_{m+1}, l_{m}\right)\right|\left\|\varphi_{n}\left(l_{m+2}\right)\right\|_{L^{2}\left(\Omega_{n}\right)}^{2} \\
& +\frac{\widetilde{\mathcal{K}}_{3} e^{\frac{\tilde{\mathcal{K}}_{2}}{\varepsilon^{\alpha}}} \int^{l_{m}} \chi_{E}\left\|\varphi_{n}(t)\right\|_{L^{2}\left(\omega \cap \Omega_{n}\right)}^{2} \mathrm{~d} t}{l_{m+1}}
\end{aligned}
$$

for each $\varepsilon>0$.

Since $l_{m}-l_{m+1}=(\kappa-1)\left(l_{1}-l\right) / \kappa^{m}$, by $(2.10)$ and $(2.8)$, we obtain

$$
\begin{aligned}
\left\|\varphi_{n}\left(l_{m}\right)\right\|_{L^{2}\left(\Omega_{n}\right)}^{2} & \leq \frac{1}{\left|E \bigcap\left(l_{m+1}, l_{m}\right)\right|} \frac{\widetilde{\mathcal{K}}_{3}}{\varepsilon^{\alpha}} e^{\frac{\tilde{\mathcal{K}}_{2}}{l_{m+1}-l m+2}} \int_{l_{m+1}}^{l_{m}} \chi_{E}\left\|\varphi_{n}(t)\right\|_{L^{2}\left(\omega \bigcap \Omega_{n}\right)}^{2} \mathrm{~d} t+\varepsilon\left\|\varphi_{n}\left(l_{m+2}\right)\right\|_{L^{2}\left(\Omega_{n}\right)}^{2} \\
& \leq \frac{3 \kappa^{m}}{\left(l_{1}-l\right)(\kappa-1)} \frac{\widetilde{\mathcal{K}}_{3}}{\varepsilon^{\alpha}} e^{\widetilde{\mathcal{K}}_{2}\left(\frac{1}{l_{1}-l} \frac{\kappa^{m+1}}{\kappa-1}\right)} \int_{l_{m+1}}^{l_{m}} \chi_{E}\left\|\varphi_{n}(t)\right\|_{L^{2}\left(\omega \bigcap \Omega_{n}\right)}^{2} \mathrm{~d} t+\varepsilon\left\|\varphi_{n}\left(l_{m+2}\right)\right\|_{L^{2}\left(\Omega_{n}\right)}^{2}
\end{aligned}
$$

for each $\varepsilon>0$. This yields

$$
\begin{aligned}
& \left\|\varphi_{n}\left(l_{m}\right)\right\|_{L^{2}\left(\Omega_{n}\right)}^{2} \\
\leq & \frac{1}{\varepsilon^{\alpha}} \frac{3}{\kappa} \frac{\widetilde{\mathcal{K}}_{3}}{\widetilde{\mathcal{K}}_{2}} e^{2 \widetilde{\mathcal{K}}_{2}\left(\frac{1}{l_{1}-l} \frac{\kappa^{m+1}}{\kappa-1}\right)} \int_{l_{m+1}}^{l_{m}} \chi_{E}\left\|\varphi_{n}(t)\right\|_{L^{2}\left(\omega \cap \Omega_{n}\right)}^{2} \mathrm{~d} t+\varepsilon\left\|\varphi_{n}\left(l_{m+2}\right)\right\|_{L^{2}\left(\Omega_{n}\right)}^{2}
\end{aligned}
$$

for each $\varepsilon>0$. Denote by $d \triangleq 2 \widetilde{\mathcal{K}}_{2} /\left[\kappa\left(l_{1}-l\right)(\kappa-1)\right]$. It follows from $(2.11)$ that

$$
\varepsilon^{\alpha} e^{-d \kappa^{m+2}}\left\|\varphi_{n}\left(l_{m}\right)\right\|_{L^{2}\left(\Omega_{n}\right)}^{2}-\varepsilon^{1+\alpha} e^{-d \kappa^{m+2}}\left\|\varphi_{n}\left(l_{m+2}\right)\right\|_{L^{2}\left(\Omega_{n}\right)}^{2} \leq \frac{3}{\kappa} \frac{\widetilde{\mathcal{K}}_{3}}{\widetilde{\mathcal{K}}_{2}} \int_{l_{m+1}}^{l_{m}} \chi_{E}\left\|\varphi_{n}(t)\right\|_{L^{2}\left(\omega \cap \Omega_{n}\right)}^{2} \mathrm{~d} t
$$


for each $\varepsilon>0$.

Choosing $\varepsilon=e^{-d \kappa^{m+2}}$ in the above inequality gives

$$
\begin{aligned}
& e^{-(1+\alpha) d \kappa^{m+2}}\left\|\varphi_{n}\left(l_{m}\right)\right\|_{L^{2}\left(\Omega_{n}\right)}^{2}-e^{-(2+\alpha) d \kappa^{m+2}}\left\|\varphi_{n}\left(l_{m+2}\right)\right\|_{L^{2}\left(\Omega_{n}\right)}^{2} \\
\leq & \frac{3}{\kappa} \frac{\widetilde{\mathcal{K}}_{3}}{\widetilde{\mathcal{K}}_{2}} \int_{l_{m+1}}^{l_{m}} \chi_{E}\left\|\varphi_{n}(t)\right\|_{L^{2}\left(\omega \cap \Omega_{n}\right)}^{2} \mathrm{~d} t .
\end{aligned}
$$

Taking $\kappa=\sqrt{(\alpha+2) /(\alpha+1)}$ in $(2.12)$, we then have

$$
e^{-(2+\alpha) d \kappa^{m}}\left\|\varphi_{n}\left(l_{m}\right)\right\|_{L^{2}\left(\Omega_{n}\right)}^{2}-e^{-(2+\alpha) d \kappa^{m+2}}\left\|\varphi_{n}\left(l_{m+2}\right)\right\|_{L^{2}\left(\Omega_{n}\right)}^{2} \leq \frac{3}{\kappa} \frac{\widetilde{\mathcal{K}}_{3}}{\widetilde{\mathcal{K}}_{2}} \int_{l_{m+1}}^{l_{m}} \chi_{E}\left\|\varphi_{n}(t)\right\|_{L^{2}\left(\omega \cap \Omega_{n}\right)}^{2} \mathrm{~d} t
$$

Changing $m$ to $2 m^{\prime}$ and summing the above inequality from $m^{\prime}=1$ to $\infty$ give the desired result. Indeed,

$$
\begin{aligned}
& e^{-2 T\|a\|_{\infty}} e^{-(2+\alpha) d \kappa^{2}}\left\|\varphi_{n}(T)\right\|_{L^{2}\left(\Omega_{n}\right)}^{2} \\
\leq & e^{-(2+\alpha) d \kappa^{2}}\left\|\varphi_{n}\left(l_{2}\right)\right\|_{L^{2}\left(\Omega_{n}\right)}^{2} \\
\leq & \sum_{m^{\prime}=1}^{\infty}\left(e^{-(2+\alpha) d \kappa^{2 m^{\prime}}}\left\|\varphi_{n}\left(l_{2 m^{\prime}}\right)\right\|_{L^{2}\left(\Omega_{n}\right)}-e^{\left.-(2+\alpha) d \kappa^{2 m^{\prime}+2}\left\|\varphi_{n}\left(l_{2 m^{\prime}+2}\right)\right\|_{L^{2}\left(\Omega_{n}\right)}^{2}\right)}\right. \\
\leq & \frac{3}{\kappa} \frac{\widetilde{\mathcal{K}}_{3}}{\widetilde{\mathcal{K}}_{2}} \sum_{m^{\prime}=1}^{\infty} \int_{l_{2 m^{\prime}+1}}^{l_{2 m^{\prime}}} \chi_{E}\left\|\varphi_{n}(t)\right\|_{L^{2}\left(\omega \cap \Omega_{n}\right)}^{2} \mathrm{~d} t \\
\leq & \frac{3}{\kappa} \frac{\widetilde{\mathcal{K}}_{3}}{\widetilde{\mathcal{K}}_{2}} \int_{0}^{T} \chi_{E}\left\|\varphi_{n}(t)\right\|_{L^{2}\left(\omega \cap \Omega_{n}\right)}^{2} \mathrm{~d} t .
\end{aligned}
$$

In summary, we finish the proof of Theorem 2.1.

\section{Proof of Theorem 1.1}

By a density argument, we can assume that $f \in C^{1}$. We will use the linearized result (i.e., Corollary 2.4) and the Kakutani-Fan-Glicksberg fixed point theorem (see, e.g., [24], Thm. 1.14) to prove Theorem 1.1.

To this end, we first define

$$
a(r) \triangleq\left\{\begin{array}{lll}
\frac{f(r)}{r} & \text { if } \quad r \neq 0 \\
f^{\prime}(0) & \text { if } \quad r=0 .
\end{array}\right.
$$

By $\left(H_{3}\right)$, we have that

$$
|a(r)| \leq L \text { for all } r \in \mathbb{R} \text {. }
$$

For each $n \geq 1$, we set

$$
\mathcal{K}_{n} \triangleq\left\{\xi \in L^{2}\left(0, T ; L^{2}\left(\Omega_{n}\right)\right) \mid\|\xi\|_{L^{2}\left(0, T ; H_{0}^{1}\left(\Omega_{n}\right)\right)}+\|\xi\|_{W^{1,2}\left(0, T ; H^{-1}\left(\Omega_{n}\right)\right)} \leq c_{0}\right\}
$$


where $c_{0}>0$ will be determined later. For each $\xi \in \mathcal{K}_{n}$, we consider the following linear equation:

$$
\left\{\begin{array}{lll}
\partial_{t} z-\Delta z+a(\xi(x, t)) z=\chi_{\omega} \cap \Omega_{n} \chi_{E} u & \text { in } & \Omega_{n} \times(0, T), \\
z=0 & \text { on } & \partial \Omega_{n} \times(0, T), \\
z(0)=z_{0} & \text { in } & \Omega_{n} .
\end{array}\right.
$$

We simply write $z(\cdot)$ for the solution of (3.2). According to Corollary 2.4, there is a positive constant $\kappa \triangleq$ $\kappa\left(r_{1}, r_{2}, E, T, L\right)$ (independent of $z_{0}, n$ and $\xi$ ) and a control $u$ so that

$$
\|u\|_{L^{2}\left(0, T ; L^{2}\left(\mathbb{R}^{N}\right)\right)} \leq \kappa\left\|z_{0}\right\|_{L^{2}\left(\mathbb{R}^{N}\right)} \text { and } z(T)=0 .
$$

For each $n \geq 1$, we next define a set-valued mapping

$$
\Phi_{n}: \mathcal{K}_{n} \rightarrow 2^{L^{2}\left(0, T ; L^{2}\left(\Omega_{n}\right)\right)}
$$

by setting

$$
\begin{array}{r}
\Phi_{n}(\xi) \triangleq\left\{z \mid \text { there exists a control } u \in L^{2}\left(0, T ; L^{2}\left(\mathbb{R}^{N}\right)\right)\right. \\
\text { so that (3.2) and (3.3) hold }\}, \quad \xi \in \mathcal{K}_{n} .
\end{array}
$$

One can easily check that $\Phi_{n}(\xi) \neq \emptyset$ for each $\xi \in \mathcal{K}_{n}$.

The rest of the proof will be organized by several steps as follows.

Step 1. We show that $\mathcal{K}_{n}$ is compact and convex in $L^{2}\left(0, T ; L^{2}\left(\Omega_{n}\right)\right)$, and that each $\Phi_{n}(\xi)$ is convex in $L^{2}\left(0, T ; L^{2}\left(\Omega_{n}\right)\right)$.

These can be directly checked.

Step 2. We claim that $\Phi_{n}\left(\mathcal{K}_{n}\right) \subset \mathcal{K}_{n}$.

Given $\xi \in \mathcal{K}_{n}$, there is a control $u$ satisfying (3.2) and (3.3). By a standard energy estimate method and by (3.1)-(3.3), we can easily check that

$$
\|z\|_{L^{2}\left(0, T ; H_{0}^{1}\left(\Omega_{n}\right)\right)}+\|z\|_{W^{1,2}\left(0, T ; H^{-1}\left(\Omega_{n}\right)\right)} \leq c_{1}\left\|z_{0}\right\|_{L^{2}\left(\Omega_{n}\right)},
$$

for a positive constant $c_{1}$ (independent of $z_{0}, n$ and $\xi$ ). Hence,

$$
z \in \mathcal{K}_{n} \text { if } c_{0}=c_{1}\left\|z_{0}\right\|_{L^{2}\left(\Omega_{n}\right)}
$$

Step 3. We show that $\operatorname{Graph}\left(\Phi_{n}\right)$ is closed.

It suffices to show that $z \in \Phi_{n}(\xi)$, provided that

$$
\xi_{\ell} \in \mathcal{K}_{n} \rightarrow \xi \text { strongly in } L^{2}\left(0, T ; L^{2}\left(\Omega_{n}\right)\right)
$$

and

$$
z_{\ell} \in \Phi_{n}\left(\xi_{\ell}\right) \rightarrow z \text { strongly in } L^{2}\left(0, T ; L^{2}\left(\Omega_{n}\right)\right) .
$$


To this end, we first observe that $\xi \in \mathcal{K}_{n}$, since $\mathcal{K}_{n}$ is convex and closed. Next we claim that there exists a subsequence of $\{\ell\}_{\ell \geq 1}$, denoted in the same manner, so that

$$
a\left(\xi_{\ell}\right) z_{\ell} \rightarrow a(\xi) z \text { strongly in } L^{2}\left(0, T ; L^{2}\left(\Omega_{n}\right)\right) .
$$

Indeed, since

$$
\xi_{\ell} \rightarrow \xi \text { strongly in } L^{2}\left(0, T ; L^{2}\left(\Omega_{n}\right)\right)
$$

we have a subsequence of $\{\ell\}_{\ell \geq 1}$, still denoted by itself, so that

$$
\xi_{\ell}(x, t) \rightarrow \xi(x, t) \text { for a.e. }(x, t) \in \Omega_{n} \times(0, T) .
$$

Then, by the definition of the function $a$, we conclude that

$$
a\left(\xi_{\ell}(x, t)\right) \rightarrow a(\xi(x, t)) \text { for a.e. }(x, t) \in \Omega_{n} \times(0, T) .
$$

By this and (3.1), we can apply the Lebesgue Dominated Convergence Theorem to obtain that

$$
\begin{aligned}
& \left\|a\left(\xi_{\ell}\right) z_{\ell}-a(\xi) z\right\|_{L^{2}\left(0, T ; L^{2}\left(\Omega_{n}\right)\right)}^{2} \\
\leq & 2\left\|a\left(\xi_{\ell}\right)\left(z_{\ell}-z\right)\right\|_{L^{2}\left(0, T ; L^{2}\left(\Omega_{n}\right)\right)}^{2}+2\left\|\left(a\left(\xi_{\ell}\right)-a(\xi)\right) z\right\|_{L^{2}\left(0, T ; L^{2}\left(\Omega_{n}\right)\right)}^{2} \\
\leq & 2 L^{2}\left\|z_{\ell}-z\right\|_{L^{2}\left(0, T ; L^{2}\left(\Omega_{n}\right)\right)}^{2}+2\left\|\left(a\left(\xi_{\ell}\right)-a(\xi)\right) z\right\|_{L^{2}\left(0, T ; L^{2}\left(\Omega_{n}\right)\right)}^{2} \\
\rightarrow & 0 .
\end{aligned}
$$

This leads to (3.4).

Finally, for each $\ell \geq 1$, since $z_{\ell} \in \Phi_{n}\left(\xi_{\ell}\right) \subset \mathcal{K}_{n}$, there is $u_{\ell} \in L^{2}\left(0, T ; L^{2}\left(\mathbb{R}^{N}\right)\right)$ with

$$
\left\|u_{\ell}\right\|_{L^{2}\left(0, T ; L^{2}\left(\mathbb{R}^{N}\right)\right)} \leq \kappa\left\|z_{0}\right\|_{L^{2}\left(\mathbb{R}^{N}\right)}
$$

so that

$$
\begin{cases}\partial_{t} z_{\ell}-\Delta z_{\ell}+a\left(\xi_{\ell}(x, t)\right) z_{\ell}=\chi_{\omega} \cap \Omega_{n} \chi_{E} u_{\ell} & \text { in } \Omega_{n} \times(0, T), \\ z_{\ell}=0 & \text { on } \partial \Omega_{n} \times(0, T) \\ z_{\ell}(0)=z_{0} & \text { in } \Omega_{n}, \\ z_{\ell}(T)=0 & \text { in } \Omega_{n}\end{cases}
$$

and

$$
\left\|z_{\ell}\right\|_{L^{2}\left(0, T ; H_{0}^{1}\left(\Omega_{n}\right)\right)}+\left\|z_{\ell}\right\|_{W^{1,2}\left(0, T ; H^{-1}\left(\Omega_{n}\right)\right)} \leq c_{0} .
$$

According to (3.5) and (3.7), there is a control $u$ and a subsequence of $\{\ell\}_{\ell \geq 1}$, denoted in the same manner, so that

$$
\begin{gathered}
u_{\ell} \rightarrow u \text { weakly in } L^{2}\left(0, T ; L^{2}\left(\mathbb{R}^{N}\right)\right), \\
z_{\ell} \rightarrow z \text { weakly in } L^{2}\left(0, T ; H_{0}^{1}\left(\Omega_{n}\right)\right) \bigcap W^{1,2}\left(0, T ; H^{-1}\left(\Omega_{n}\right)\right),
\end{gathered}
$$


and

$$
z_{\ell}(T) \rightarrow z(T) \text { strongly in } L^{2}\left(\Omega_{n}\right)
$$

Passing to the limit for $\ell \rightarrow+\infty$ in (3.5) and (3.6), making use of (3.4) and (3.8)-(3.10), we obtain that $z \in \Phi_{n}(\xi)$.

Step 4. We apply the Kakutani-Fan-Glicksberg Theorem to end the proof.

From the conclusions in the above three steps, we find that the map $\Phi_{n}$ satisfies conditions of the KakutaniFan-Glicksberg Theorem. Thus we can apply this theorem to conclude that there exists $z \in \mathcal{K}_{n}$ so that $z \in \Phi_{n}(z)$. Then, by the definition of $\Phi_{n}$ and using the fact that

$$
a(z(x, t)) z(x, t)=f(z(x, t))
$$

one can finish the proof of Theorem 1.1.

\section{Proof of Theorem 1.5}

For the sake of completeness ${ }^{1}$, we first consider the well-posedness of the following non-homogeneous semilinear heat equation in $\mathbb{R}^{N}$ :

$$
\left\{\begin{array}{lll}
\partial_{t} y-\Delta y+f(y)=g & \text { in } \mathbb{R}^{N} \times(0, T) \\
y(0)=y_{0} & \text { in } \mathbb{R}^{N}
\end{array}\right.
$$

where $y_{0} \in L^{2}\left(\mathbb{R}^{N}\right), g \in L^{2}\left(\mathbb{R}^{N} \times(0, T)\right)$, and the nonlinearity $f$ satisfies the assumption $\left(H_{3}\right)$.

Definition 4.1. We say that $y$ is a weak solution of (4.1) if

(i) $y \in L^{2}\left(0, T ; H^{1}\left(\mathbb{R}^{N}\right)\right) \bigcap W^{1,2}\left(0, T ; H^{-1}\left(\mathbb{R}^{N}\right)\right) \subset C\left([0, T] ; L^{2}\left(\mathbb{R}^{N}\right)\right)$ and $y(0)=y_{0}$;

(ii) For each $\psi \in H^{1}\left(\mathbb{R}^{N}\right)$, the following equality holds:

$$
\left\langle\partial_{t} y(t), \psi\right\rangle_{H^{-1}\left(\mathbb{R}^{N}\right), H^{1}\left(\mathbb{R}^{N}\right)}+\langle\nabla y(t), \nabla \psi\rangle_{L^{2}\left(\mathbb{R}^{N}\right)}+\langle f(y(t)), \psi\rangle_{L^{2}\left(\mathbb{R}^{N}\right)}=\langle g(t), \psi\rangle_{L^{2}\left(\mathbb{R}^{N}\right)}
$$

for a.e. $t \in(0, T)$.

Before presenting the proof of Theorem 1.5, we first state the well-posedness of (4.1) in the sense of the above definition.

Lemma 4.2. Under the assumption $\left(H_{3}\right)$, for each $y_{0} \in L^{2}\left(\mathbb{R}^{N}\right)$ and each $g \in L^{2}\left(0, T ; L^{2}\left(\mathbb{R}^{N}\right)\right)$, the equation (4.1) has a unique weak solution.

Remark 4.3. The argument below is also effective in our proof of Theorem 1.5. Indeed, it is inspired by the approach in Section 4.4 of [22] constructing weak solutions for the Navier-Stokes equations on the whole space. The main idea there is to use solutions of the Navier-Stokes equations on a sequence of expanding bounded domains as a sequence of approximate solutions on the whole space and show the convergence of such solutions.

Proof. For each $n \geq 1$, we shall consider the equation

$$
\begin{cases}\partial_{t} y_{n}-\Delta y_{n}+f\left(y_{n}\right)=g & \text { in } \Omega_{n} \times(0, T) \\ y_{n}=0 & \text { on } \partial \Omega_{n} \times(0, T) \\ y_{n}(0)=y_{0} & \text { in } \Omega_{n}\end{cases}
$$

\footnotetext{
${ }^{1}$ Although we believe that the existence and uniqueness of weak solutions for nonlinear heat equations in $\mathbb{R}^{N}$ have been well established in the literature, we did not yet find the exact reference with precise proofs.
} 
where $\Omega_{n}$ is constructed as in $\left(H_{2}\right)$. By $\left(H_{3}\right),(4.2)$, and a standard energy estimate method, we can easily check that

$$
\left\|y_{n}\right\|_{L^{2}\left(0, T ; H_{0}^{1}\left(\Omega_{n}\right)\right)}+\left\|y_{n}\right\|_{W^{1,2}\left(0, T ; H^{-1}\left(\Omega_{n}\right)\right)} \leq C .
$$

Here and throughout the proof of this lemma, $C$ denotes a positive constant independent of $n$.

We extend $y_{n}$ to $\mathbb{R}^{N} \times(0, T)$ by zero and still denote this extension by $y_{n}$. On one hand, by (4.3), there is a subsequence of $\{n\}_{n \geq 1}$, still denoted by itself, and $y^{*} \in L^{2}\left(0, T ; H^{1}\left(\mathbb{R}^{N}\right)\right)$, so that

$$
y_{n} \rightarrow y^{*} \text { weakly in } L^{2}\left(0, T ; H^{1}\left(\mathbb{R}^{N}\right)\right) \text {. }
$$

On the other hand, for each $M>0$, there exists a positive integer $n_{0}(M)$ so that

$$
H^{-1}\left(\Omega_{n}\right) \subset H^{-1}\left(B_{M}(0)\right) \text { and }\|\cdot\|_{H^{-1}\left(B_{M}(0)\right)} \leq\|\cdot\|_{H^{-1}\left(\Omega_{n}\right)} \text { for all } n \geq n_{0}(M) .
$$

These, along with (4.3) and (4.4), imply that $y^{*} \in W^{1,2}\left(0, T ; H^{-1}\left(B_{M}(0)\right)\right)$ and there is a subsequence of $\{n\}_{n \geq 1}$, denoted in the same manner, so that

$$
y_{n} \rightarrow y^{*} \text { weakly in } L^{2}\left(0, T ; H^{1}\left(\mathbb{R}^{N}\right)\right) \bigcap W^{1,2}\left(0, T ; H^{-1}\left(B_{M}(0)\right)\right) \text {, }
$$

and

$$
y_{n} \rightarrow y^{*} \text { strongly in } L^{2}\left(0, T ; L^{2}\left(B_{M}(0)\right)\right) \text {. }
$$

It follows from $\left(H_{3}\right)$ and (4.6) that

$$
f\left(y_{n}\right) \rightarrow f\left(y^{*}\right) \text { strongly in } L^{2}\left(0, T ; L^{2}\left(B_{M}(0)\right)\right)
$$

Arbitrarily fix $\varphi \in C_{0}^{\infty}\left(\mathbb{R}^{N} \times(0, T)\right)$. Let $M>0$ be large enough so that the support of $\varphi$ is contained in $B_{M}(0) \times(0, T)$. Then for all $n \geq n_{0}(M)$, we have that

$$
\begin{aligned}
& -\int_{0}^{T}\left\langle y_{n}, \partial_{t} \varphi\right\rangle_{L^{2}\left(B_{M}(0)\right)} \mathrm{d} t+\int_{0}^{T}\left\langle\nabla y_{n}, \nabla \varphi\right\rangle_{L^{2}\left(B_{M}(0)\right)} \mathrm{d} t+\int_{0}^{T}\left\langle f\left(y_{n}\right), \varphi\right\rangle_{L^{2}\left(B_{M}(0)\right)} \mathrm{d} t \\
= & \int_{0}^{T}\langle g, \varphi\rangle_{L^{2}\left(B_{M}(0)\right)} \mathrm{d} t .
\end{aligned}
$$

Passing to the limit for $n \rightarrow \infty$ in (4.8), by (4.5) and (4.7), we obtain that

$$
\begin{aligned}
& -\int_{0}^{T} \int_{\mathbb{R}^{N}} y^{*} \partial_{t} \varphi \mathrm{d} x \mathrm{~d} t+\int_{0}^{T} \int_{\mathbb{R}^{N}} \nabla y^{*} \cdot \nabla \varphi \mathrm{d} x \mathrm{~d} t+\int_{0}^{T} \int_{\mathbb{R}^{N}} f\left(y^{*}\right) \varphi \mathrm{d} x \mathrm{~d} t \\
= & \int_{0}^{T} \int_{\mathbb{R}^{N}} g \varphi \mathrm{d} x \mathrm{~d} t,
\end{aligned}
$$

which indicates that

$$
\partial_{t} y^{*}=\Delta y^{*}-f\left(y^{*}\right)+g \text { in the sense of distribution. }
$$


Since $\Delta y^{*}-f\left(y^{*}\right)+g \in L^{2}\left(0, T ; H^{-1}\left(\mathbb{R}^{N}\right)\right)$, it follows from (4.9) that $\partial_{t} y^{*} \in L^{2}\left(0, T ; H^{-1}\left(\mathbb{R}^{N}\right)\right)$. Hence,

$$
y^{*} \in L^{2}\left(0, T ; H^{1}\left(\mathbb{R}^{N}\right)\right) \bigcap W^{1,2}\left(0, T ; H^{-1}\left(\mathbb{R}^{N}\right)\right) \subset C\left([0, T] ; L^{2}\left(\mathbb{R}^{N}\right)\right) .
$$

This, along with (4.9) and a density argument, implies that for each $\psi \in H^{1}\left(\mathbb{R}^{N}\right)$,

$$
\left\langle\partial_{t} y^{*}(t), \psi\right\rangle_{H^{-1}\left(\mathbb{R}^{N}\right), H^{1}\left(\mathbb{R}^{N}\right)}+\left\langle\nabla y^{*}(t), \nabla \psi\right\rangle_{L^{2}\left(\mathbb{R}^{N}\right)}+\left\langle f\left(y^{*}(t)\right), \psi\right\rangle_{L^{2}\left(\mathbb{R}^{N}\right)}=\langle g(t), \psi\rangle_{L^{2}\left(\mathbb{R}^{N}\right)}
$$

for a.e. $t \in(0, T)$.

We next show that

$$
y^{*}(0)=y_{0} .
$$

To this end, we arbitrarily fix $\psi \in C_{0}^{\infty}\left(\mathbb{R}^{N}\right)$. Let $M>0$ be large enough so that the support of $\psi$ is contained in $B_{M}(0)$. Set $\varphi(x, t) \triangleq(T-t) \psi(x) / T$. Then for all $n \geq n_{0}(M)$, multiplying both sides of (4.2) by $\varphi$ and integrating it over $\mathbb{R}^{N} \times(0, T)$, we obtain that

$$
\begin{aligned}
& -\int_{0}^{T}\left\langle y_{n}, \partial_{t} \varphi\right\rangle_{L^{2}\left(B_{M}(0)\right)} \mathrm{d} t+\int_{0}^{T}\left\langle\nabla y_{n}, \nabla \varphi\right\rangle_{L^{2}\left(B_{M}(0)\right)} \mathrm{d} t+\int_{0}^{T}\left\langle f\left(y_{n}\right), \varphi\right\rangle_{L^{2}\left(B_{M}(0)\right)} \mathrm{d} t \\
= & \int_{0}^{T^{T}}\langle g, \varphi\rangle_{L^{2}\left(B_{M}(0)\right)} \mathrm{d} t+\left\langle y_{0}, \psi\right\rangle_{L^{2}\left(B_{M}(0)\right)} .
\end{aligned}
$$

Passing to the limit for $n \rightarrow \infty$ in (4.13), by (4.5) and (4.7), we obtain that

$$
\begin{aligned}
& -\int_{0}^{T}\left\langle y^{*}, \partial_{t} \varphi\right\rangle_{L^{2}\left(\mathbb{R}^{N}\right)} \mathrm{d} t+\int_{0}^{T}\left\langle\nabla y^{*}, \nabla \varphi\right\rangle_{L^{2}\left(\mathbb{R}^{N}\right)} \mathrm{d} t+\int_{0}^{T}\left\langle f\left(y^{*}\right), \varphi\right\rangle_{L^{2}\left(\mathbb{R}^{N}\right)} \mathrm{d} t \\
= & \int_{0}^{T}\langle g, \varphi\rangle_{L^{2}\left(\mathbb{R}^{N}\right)} \mathrm{d} t+\left\langle y_{0}, \psi\right\rangle_{L^{2}\left(\mathbb{R}^{N}\right)} .
\end{aligned}
$$

Moreover, multiplying both sides of $(4.11)$ by $(T-t) / T$ and integrating it over $(0, T)$, we have that

$$
\begin{aligned}
& -\int_{0}^{T}\left\langle y^{*}, \partial_{t} \varphi\right\rangle_{L^{2}\left(\mathbb{R}^{N}\right)} \mathrm{d} t+\int_{0}^{T}\left\langle\nabla y^{*}, \nabla \varphi\right\rangle_{L^{2}\left(\mathbb{R}^{N}\right)} \mathrm{d} t+\int_{0}^{T}\left\langle f\left(y^{*}\right), \varphi\right\rangle_{L^{2}\left(\mathbb{R}^{N}\right)} \mathrm{d} t \\
= & \int_{0}^{T}\langle g, \varphi\rangle_{L^{2}\left(\mathbb{R}^{N}\right)} \mathrm{d} t+\left\langle y^{*}(0), \psi\right\rangle_{L^{2}\left(\mathbb{R}^{N}\right)} .
\end{aligned}
$$

It follows from (4.14) and (4.15) that

$$
\left\langle y^{*}(0)-y_{0}, \psi\right\rangle_{L^{2}\left(\mathbb{R}^{N}\right)}=0 \text { for each } \psi \in C_{0}^{\infty}\left(\mathbb{R}^{N}\right),
$$

which indicates (4.12).

By (4.10)-(4.12) and Definition 4.1, we see that $y^{*}$ is a weak solution of (4.1). Finally, we show the uniqueness of the weak solution. WLOG, we assume that $\widetilde{y}$ is also a weak solution of (4.1). According to Definition 4.1, it holds that

$$
\begin{aligned}
& \left\langle\partial_{t}\left(y^{*}-\widetilde{y}\right)(t),\left(y^{*}-\widetilde{y}\right)(t)\right\rangle_{H^{-1}\left(\mathbb{R}^{N}\right), H^{1}\left(\mathbb{R}^{N}\right)} \\
& +\left\langle\nabla\left(y^{*}-\widetilde{y}\right)(t), \nabla\left(y^{*}-\widetilde{y}\right)(t)\right\rangle_{L^{2}\left(\mathbb{R}^{N}\right)}+\left\langle f\left(y^{*}(t)\right)-f(\widetilde{y}(t)),\left(y^{*}-\widetilde{y}\right)(t)\right\rangle_{L^{2}\left(\mathbb{R}^{N}\right)}=0
\end{aligned}
$$


for a.e. $t \in(0, T)$, and $y^{*}(0)=\widetilde{y}(0)=y_{0}$. Integrating $(4.16)$ over $(0, t)$, we obtain that

$$
\left\|\left(y^{*}-\widetilde{y}\right)(t)\right\|_{L^{2}\left(\mathbb{R}^{N}\right)}^{2} \leq 2 L \int_{0}^{t}\left\|\left(y^{*}-\widetilde{y}\right)(s)\right\|_{L^{2}\left(\mathbb{R}^{N}\right)}^{2} \mathrm{~d} s .
$$

By Gronwall's inequality, we obtain from the latter inequality that $y^{*}=\widetilde{y}$.

In summary, we finish the proof of Lemma 4.2.

Now, we are able to present the proof of Theorem 1.5.

Proof of Theorem 1.5. For each $n \geq 1$, according to Theorem 1.1, there is a control $u_{n} \in L^{2}\left(0, T ; L^{2}\left(\mathbb{R}^{N}\right)\right)$ so that

$$
\begin{gathered}
\begin{cases}\partial_{t} y_{n}-\Delta y_{n}+f\left(y_{n}\right)=\chi_{\omega} \chi_{E} u_{n} & \text { in } \Omega_{n} \times(0, T) \\
y_{n}=0 & \text { on } \partial \Omega_{n} \times(0, T) \\
y_{n}(0)=y_{0} & \text { in } \Omega_{n},\end{cases} \\
\qquad u_{n}\left\|_{L^{2}\left(0, T ; L^{2}\left(\mathbb{R}^{N}\right)\right)} \leq \kappa\right\| y_{0} \|_{L^{2}\left(\mathbb{R}^{N}\right)}
\end{gathered}
$$

and

$$
y_{n}(T)=0 \text { in } \Omega_{n}
$$

By a standard energy estimate method, $\left(H_{3}\right),(4.17)$ and $(4.18)$, we can easily check that

$$
\left\|y_{n}\right\|_{L^{2}\left(0, T ; H_{0}^{1}\left(\Omega_{n}\right)\right)}+\left\|y_{n}\right\|_{W^{1,2}\left(0, T ; H^{-1}\left(\Omega_{n}\right)\right)} \leq C,
$$

where $C$ is a positive constant independent of $n$.

We extend $y_{n}$ to $\mathbb{R}^{N} \times(0, T)$ by 0 and still denote this extension by $y_{n}$. By (4.20) and (4.18), there is a subsequence of $\{n\}_{n \geq 1}$, still denoted by itself, and $\left(u^{*}, y^{*}\right) \in L^{2}\left(0, T ; L^{2}\left(\mathbb{R}^{N}\right)\right) \times L^{2}\left(0, T ; H^{1}\left(\mathbb{R}^{N}\right)\right)$, so that

$$
y_{n} \rightarrow y^{*} \text { weakly in } L^{2}\left(0, T ; H^{1}\left(\mathbb{R}^{N}\right)\right)
$$

and

$$
u_{n} \rightarrow u^{*} \text { weakly in } L^{2}\left(0, T ; L^{2}\left(\mathbb{R}^{N}\right)\right) \text {. }
$$

On one hand, by similar arguments as those in Lemma 4.2, we observe that $y^{*}$ is the unique weak solution of (1.2) (where $u$ is replaced by $u^{*}$ ), and for each $M>0$, there is a subsequence of $\{n\}_{n \geq 1}$, denoted in the same manner, so that

$$
f\left(y_{n}\right) \rightarrow f\left(y^{*}\right) \text { strongly in } L^{2}\left(0, T ; L^{2}\left(B_{M}(0)\right)\right)
$$

On the other hand, we arbitrarily fix $\psi \in C_{0}^{\infty}\left(\mathbb{R}^{N}\right)$. Let $M>0$ be large enough so that the support of $\psi$ is contained in $B_{M}(0)$. Set $\varphi(x, t) \triangleq t \psi(x) / T$. Then for all $n \geq n_{0}(M)$ (where $n_{0}(M)$ is the same integer as that 
in Lem. 4.2), multiplying both sides of (4.17) by $\varphi$ and integrating it over $\mathbb{R}^{N} \times(0, T)$, we obtain that

$$
\begin{aligned}
& -\int_{0}^{T}\left\langle y_{n}, \partial_{t} \varphi\right\rangle_{L^{2}\left(B_{M}(0)\right)} \mathrm{d} t+\int_{0}^{T}\left\langle\nabla y_{n}, \nabla \varphi\right\rangle_{L^{2}\left(B_{M}(0)\right)} \mathrm{d} t+\int_{0}^{T}\left\langle f\left(y_{n}\right), \varphi\right\rangle_{L^{2}\left(B_{M}(0)\right)} \mathrm{d} t \\
= & \int_{0}^{T}\left\langle\chi_{\omega} \chi_{E} u_{n}, \varphi\right\rangle_{L^{2}\left(B_{M}(0)\right)} \mathrm{d} t-\left\langle y_{n}(T), \psi\right\rangle_{L^{2}\left(B_{M}(0)\right)} .
\end{aligned}
$$

Passing to the limit for $n \rightarrow \infty$ in (4.24), by (4.19) and (4.21)-(4.23), we obtain that

$$
\begin{aligned}
& -\int_{0}^{T}\left\langle y^{*}, \partial_{t} \varphi\right\rangle_{L^{2}\left(\mathbb{R}^{N}\right)} \mathrm{d} t+\int_{0}^{T}\left\langle\nabla y^{*}, \nabla \varphi\right\rangle_{L^{2}\left(\mathbb{R}^{N}\right)} \mathrm{d} t+\int_{0}^{T}\left\langle f\left(y^{*}\right), \varphi\right\rangle_{L^{2}\left(\mathbb{R}^{N}\right)} \mathrm{d} t \\
= & \int_{0}^{T}\left\langle\chi_{\omega} \chi_{E} u^{*}, \varphi\right\rangle_{L^{2}\left(\mathbb{R}^{N}\right)} \mathrm{d} t .
\end{aligned}
$$

Moreover, since $y^{*}$ is the weak solution of (1.2) (where $u$ is replaced by $u^{*}$ ), we have that

$$
\begin{aligned}
& -\int_{0}^{T}\left\langle y^{*}, \partial_{t} \varphi\right\rangle_{L^{2}\left(\mathbb{R}^{N}\right)} \mathrm{d} t+\int_{0}^{T}\left\langle\nabla y^{*}, \nabla \varphi\right\rangle_{L^{2}\left(\mathbb{R}^{N}\right)} \mathrm{d} t+\int_{0}^{T}\left\langle f\left(y^{*}\right), \varphi\right\rangle_{L^{2}\left(\mathbb{R}^{N}\right)} \mathrm{d} t \\
= & \int_{0}^{T}\left\langle\chi_{\omega} \chi_{E} u^{*}, \varphi\right\rangle_{L^{2}\left(\mathbb{R}^{N}\right)} \mathrm{d} t-\left\langle y^{*}(T), \psi\right\rangle_{L^{2}\left(\mathbb{R}^{N}\right)} .
\end{aligned}
$$

It follows from (4.25) and (4.26) that $\left\langle y^{*}(T), \psi\right\rangle_{L^{2}\left(\mathbb{R}^{N}\right)}=0$ for each $\psi \in C_{0}^{\infty}\left(\mathbb{R}^{N}\right)$. This implies that $y^{*}(T)=0$.

Hence, we finish the proof of Theorem 1.5.

\section{Appendix A.}

First of all, we give two standard energy estimates for solutions of (2.1). Their proofs are adapted from Lemmas 2.1 and 2.2 in [8], respectively.

Lemma A.1. There is a constant $\widetilde{C}_{1}>1$ so that for any $\varphi_{0} \in L^{2}\left(\Omega_{n}\right)$, the solution $\varphi_{n}$ of (2.1) satisfies

$$
\begin{aligned}
& \max _{t \in\left[T-\tau_{1}, T\right]} \int_{B_{r}\left(x_{0}\right) \cap \Omega_{n}} \varphi_{n}^{2}(x, t) \mathrm{d} x+\int_{T-\tau_{1}}^{T} \int_{B_{r}\left(x_{0}\right) \cap \Omega_{n}}\left|\nabla \varphi_{n}(x, s)\right|^{2} \mathrm{~d} x \mathrm{~d} s \\
\leq & \widetilde{C}_{1}\left[(R-r)^{-2}+\left(\tau_{2}-\tau_{1}\right)^{-1}+\|a\|_{\infty}\right] \int_{T-\tau_{2}}^{T} \int_{B_{R}\left(x_{0}\right) \cap \Omega_{n}} \varphi_{n}^{2}(x, s) \mathrm{d} x \mathrm{~d} s,
\end{aligned}
$$

for all $0<r<R<+\infty, 0<\tau_{1}<\tau_{2}<T$ and $x_{0} \in \Omega_{n}$.

Proof. For simplicity we write $B_{r} \triangleq B_{r}\left(x_{0}\right)$ and $B_{R} \triangleq B_{R}\left(x_{0}\right)$. Let $\eta \in C_{0}^{\infty}\left(B_{R}\right)$ verify

$$
0 \leq \eta(\cdot) \leq 1 \text { in } B_{R}, \eta(\cdot)=1 \text { in } B_{r} \text { and }|\nabla \eta(\cdot)| \leq C(R-r)^{-1} .
$$

Here and throughout the proof of Lemma A.1, $C$ denotes a generic positive constant. Let $\xi \in C^{\infty}(\mathbb{R})$ satisfy

$$
\begin{gathered}
0 \leq \xi(\cdot) \leq 1,\left|\xi^{\prime}(\cdot)\right| \leq C\left(\tau_{2}-\tau_{1}\right)^{-1} \text { in } \mathbb{R}, \\
\xi(\cdot)=0 \text { in }\left(-\infty, T-\tau_{2}\right] \text { and } \xi(\cdot)=1 \text { in }\left[T-\tau_{1},+\infty\right) .
\end{gathered}
$$


Multiplying the first equation of $(2.1)$ by $\eta^{2} \xi^{2} \varphi_{n}$ and integrating it over $\left(B_{R} \bigcap \Omega_{n}\right) \times\left(T-\tau_{2}, t\right)$ for $t \in\left[T-\tau_{1}, T\right]$, we obtain

$$
\begin{aligned}
& \frac{1}{2} \int_{B_{R} \cap \Omega_{n}} \eta^{2} \xi^{2}(t) \varphi_{n}^{2}(x, t) \mathrm{d} x+\int_{T-\tau_{2}}^{t} \int_{B_{R} \cap \Omega_{n}} \eta^{2} \xi^{2}\left|\nabla \varphi_{n}\right|^{2} \mathrm{~d} x \mathrm{~d} s \\
= & -2 \int_{T-\tau_{2}}^{t} \int_{B_{R} \cap \Omega_{n}} \xi^{2} \eta \varphi_{n} \nabla \eta \cdot \nabla \varphi_{n} \mathrm{~d} x \mathrm{~d} s+\int_{T-\tau_{2}}^{t} \int_{B_{R} \cap \Omega_{n}} \eta^{2} \xi \xi^{\prime} \varphi_{n}^{2} \mathrm{~d} x \mathrm{~d} s \\
& -\int_{T-\tau_{2}}^{t} \int_{B_{R} \cap \Omega_{n}} a \eta^{2} \xi^{2} \varphi_{n}^{2} \mathrm{~d} x \mathrm{~d} s .
\end{aligned}
$$

Applying Young's inequality to the first term on the right hand of (A.5), we have

$$
\begin{aligned}
& \int_{B_{R} \cap \Omega_{n}} \eta^{2} \xi^{2}(t) \varphi_{n}^{2}(x, t) \mathrm{d} x+\int_{T-\tau_{2}}^{t} \int_{B_{R} \cap \Omega_{n}} \eta^{2} \xi^{2}\left|\nabla \varphi_{n}\right|^{2} \mathrm{~d} x \mathrm{~d} s \\
\leq & 4 \int_{T-\tau_{2}}^{t} \int_{B_{R} \cap \Omega_{n}}|\nabla \eta|^{2} \xi^{2} \varphi_{n}^{2} \mathrm{~d} x \mathrm{~d} s+2 \int_{T-\tau_{2}}^{t} \int_{B_{R} \cap \Omega_{n}} \eta^{2} \xi \xi^{\prime} \varphi_{n}^{2} \mathrm{~d} x \mathrm{~d} s \\
& -2 \int_{T-\tau_{2}}^{t} \int_{B_{R} \cap \Omega_{n}} a \eta^{2} \xi^{2} \varphi_{n}^{2} \mathrm{~d} x \mathrm{~d} s .
\end{aligned}
$$

This, along with (A.2)-(A.4), implies that

$$
\begin{aligned}
& \int_{B_{r} \cap \Omega_{n}} \varphi_{n}^{2}(x, t) \mathrm{d} x+\int_{T-\tau_{1}}^{t} \int_{B_{r} \cap \Omega_{n}}\left|\nabla \varphi_{n}\right|^{2} \mathrm{~d} x \mathrm{~d} s \\
\leq & C\left[(R-r)^{-2}+\left(\tau_{2}-\tau_{1}\right)^{-1}+\|a\|_{\infty}\right] \int_{T-\tau_{2}}^{T} \int_{B_{R} \cap \Omega_{n}} \varphi_{n}^{2} \mathrm{~d} x \mathrm{~d} s \text { for each } t \in\left[T-\tau_{1}, T\right] .
\end{aligned}
$$

Hence, (A.1) follows from the last inequality immediately.

Lemma A.2. There is a constant $\widetilde{C}_{2}>0$ so that for any $\varphi_{0} \in L^{2}\left(\Omega_{n}\right)$, the solution $\varphi_{n}$ of (2.1) satisfies

$$
\max _{t \in[T-\tau, T]} \int_{B_{R}\left(x_{0}\right) \cap \Omega_{n}}\left|\nabla \varphi_{n}(x, t)\right|^{2} \mathrm{~d} x \leq \widetilde{C}_{2}\left(R^{-4}+\tau^{-2}+\|a\|_{\infty}^{2}\right) \int_{T-2 \tau}^{T} \int_{B_{2 R}\left(x_{0}\right) \cap \Omega_{n}} \varphi_{n}^{2}(x, s) \mathrm{d} x \mathrm{~d} s,
$$

for all $0<R<+\infty, 0<\tau<T / 2$ and $x_{0} \in \Omega_{n}$.

Proof. For each $r^{\prime}>0$, we write $B_{r^{\prime}} \triangleq B_{r^{\prime}}\left(x_{0}\right)$. Let $\eta \in C_{0}^{\infty}\left(B_{4 R / 3}\right)$ satisfy

$$
0 \leq \eta(\cdot) \leq 1,|\nabla \eta(\cdot)| \leq C R^{-1},|\Delta \eta(\cdot)| \leq C R^{-2} \text { in } B_{4 R / 3}
$$

and

$$
\eta(\cdot)=1 \text { in } B_{R}
$$

Here and throughout the proof of Lemma A.2, $C$ denotes a generic positive constant. Let $\xi \in C^{\infty}(\mathbb{R})$ verify

$$
0 \leq \xi(\cdot) \leq 1,\left|\xi^{\prime}(\cdot)\right| \leq C \tau^{-1} \text { in } \mathbb{R},
$$




$$
\xi(\cdot)=0 \text { in }(-\infty, T-4 \tau / 3] \text { and } \xi(\cdot)=1 \text { in }[T-\tau,+\infty) .
$$

Denote by $z \triangleq \eta \xi \varphi_{n}$. It is easy to check that

$$
\begin{cases}\partial_{t} z-\Delta z=\left(\eta \xi^{\prime}-\xi \Delta \eta-a \eta \xi\right) \varphi_{n}-2 \xi \nabla \eta \cdot \nabla \varphi_{n} & \text { in }\left(B_{4 R / 3} \bigcap \Omega_{n}\right) \times(0, T), \\ z=0 & \text { on } \partial\left(B_{4 R / 3} \bigcap \Omega_{n}\right) \times(0, T), \\ z(T-4 \tau / 3)=0 & \text { in } B_{4 R / 3} \bigcap \Omega_{n}\end{cases}
$$

On one hand, for each $t \in[T-\tau, T]$, we have

$$
-2 \int_{T-4 \tau / 3}^{t} \int_{B_{4 R / 3} \cap \Omega_{n}} \Delta z \partial_{s} z \mathrm{~d} x \mathrm{~d} s=\int_{B_{4 R / 3} \cap \Omega_{n}}|\nabla z(x, t)|^{2} \mathrm{~d} x-\int_{B_{4 R / 3} \cap \Omega_{n}}|\nabla z(x, T-4 \tau / 3)|^{2} \mathrm{~d} x,
$$

which indicates

$$
\int_{B_{4 R / 3} \cap \Omega_{n}}|\nabla z(x, t)|^{2} \mathrm{~d} x \leq \int_{T-4 \tau / 3}^{t} \int_{B_{4 R / 3} \cap \Omega_{n}}\left(\Delta z-\partial_{s} z\right)^{2} \mathrm{~d} x \mathrm{~d} s \text { for each } t \in[T-\tau, T] .
$$

This, along with (A.8) and the second relation of (A.10), implies that

$$
\max _{t \in[T-\tau, T]} \int_{B_{R} \cap \Omega_{n}}\left|\nabla \varphi_{n}(x, t)\right|^{2} \mathrm{~d} x \leq \int_{T-4 \tau / 3}^{T} \int_{B_{4 R / 3} \cap \Omega_{n}}\left(\Delta z-\partial_{s} z\right)^{2} \mathrm{~d} x \mathrm{~d} s .
$$

On the other hand,

$$
\begin{aligned}
& \int_{T-4 \tau / 3}^{T} \int_{B_{4 R / 3} \cap \Omega_{n}}\left[\left(\eta \xi^{\prime}-\xi \Delta \eta-a \eta \xi\right) \varphi_{n}-2 \xi \nabla \eta \cdot \nabla \varphi_{n}\right]^{2} \mathrm{~d} x \mathrm{~d} t \\
\leq & 8 \int_{T-4 \tau / 3}^{T} \int_{B_{4 R / 3} \cap \Omega_{n}}\left[\left(\eta^{2}\left|\xi^{\prime}\right|^{2}+\xi^{2}|\Delta \eta|^{2}+a^{2} \eta^{2} \xi^{2}\right) \varphi_{n}^{2}+\xi^{2}|\nabla \eta|^{2}\left|\nabla \varphi_{n}\right|^{2}\right] \mathrm{d} x \mathrm{~d} t .
\end{aligned}
$$

By (A.13), (A.7) and (A.9), we get

$$
\begin{aligned}
& \int_{T-4 \tau / 3}^{T} \int_{B_{4 R / 3} \cap \Omega_{n}}\left[\left(\eta \xi^{\prime}-\xi \Delta \eta-a \eta \xi\right) \varphi_{n}-2 \xi \nabla \eta \cdot \nabla \varphi_{n}\right]^{2} \mathrm{~d} x \mathrm{~d} t \\
\leq & C\left(\tau^{-2}+R^{-4}+\|a\|_{\infty}^{2}\right) \int_{T-4 \tau / 3}^{T} \int_{B_{4 R / 3} \cap \Omega_{n}} \varphi_{n}^{2} \mathrm{~d} x \mathrm{~d} t \\
& +C R^{-2} \int_{T-4 \tau / 3}^{T} \int_{B_{4 R / 3} \cap \Omega_{n}}\left|\nabla \varphi_{n}\right|^{2} \mathrm{~d} x \mathrm{~d} t .
\end{aligned}
$$

According to (A.1) (where $r, R, \tau_{1}$ and $\tau_{2}$ are replaced by $4 R / 3,2 R, 4 \tau / 3$ and $2 \tau$, respectively), it is clear that

$$
\int_{T-4 \tau / 3}^{T} \int_{B_{4 R / 3} \cap \Omega_{n}}\left|\nabla \varphi_{n}\right|^{2} \mathrm{~d} x \mathrm{~d} t \leq C\left(\tau^{-1}+R^{-2}+\|a\|_{\infty}\right) \int_{T-2 \tau}^{T} \int_{B_{2 R} \cap \Omega_{n}} \varphi_{n}^{2} \mathrm{~d} x \mathrm{~d} t .
$$


This, along with (A.14), implies that

$$
\begin{aligned}
& \int_{T-4 \tau / 3}^{T} \int_{B_{4 R / 3} \cap \Omega_{n}}\left[\left(\eta \xi^{\prime}-\xi \Delta \eta-a \eta \xi\right) \varphi_{n}-2 \xi \nabla \eta \cdot \nabla \varphi_{n}\right]^{2} \mathrm{~d} x \mathrm{~d} t \\
\leq & C\left(\tau^{-2}+R^{-4}+\|a\|_{\infty}^{2}\right) \int_{T-2 \tau}^{T} \int_{B_{2 R} \cap \Omega_{n}} \varphi_{n}^{2} \mathrm{~d} x \mathrm{~d} t \\
& +C R^{-2}\left(\tau^{-1}+R^{-2}+\|a\|_{\infty}\right) \int_{T-2 \tau}^{T} \int_{B_{2 R} \cap \Omega_{n}} \varphi_{n}^{2} \mathrm{~d} x \mathrm{~d} t \\
\leq & C\left(\tau^{-2}+R^{-4}+\|a\|_{\infty}^{2}\right) \int_{T-2 \tau}^{T} \int_{B_{2 R} \cap \Omega_{n}} \varphi_{n}^{2} \mathrm{~d} x \mathrm{~d} t .
\end{aligned}
$$

Hence, (A.6) follows from the last inequality, (A.12) and the first equation of (A.11).

In order to give the proof of Proposition 2.5, we need the following auxiliary lemma, which is similar to Lemma 2.3 of [8] just replacing $B_{r}\left(x_{0}\right)$ by $B_{r}\left(x_{0}\right) \cap \Omega_{n}$ in (A.15) below.

Lemma A.3. Let $0<2 r \leq R<+\infty$ and $\delta \in(0,1]$. Then there are two constants $\widetilde{C}_{3} \triangleq \widetilde{C}_{3}(r, \delta)>0$ and $\widetilde{C}_{4} \triangleq \widetilde{C}_{4}(r, \delta)>0$ so that for any $0<\tau_{1}<\tau_{2}<T, x_{0} \in \Omega_{n}, \varphi_{0} \in L^{2}\left(\Omega_{n}\right)$ with $\varphi_{0} \neq 0$, the quantity

$$
h_{0}=\frac{\widetilde{C}_{3}}{\ln \left[\left(1+\widetilde{C}_{4}\right)\left(e^{\left[1+2 \widetilde{C}_{1}\left(1+\frac{1}{r^{2}}\right)\right]\left(1+\frac{1}{\tau_{2}-\tau_{1}}+\|a\|_{\infty}^{2 / 3}\right)+\frac{4 \widetilde{C}_{3}}{T}+2 T\|a\|_{\infty}}\right) \frac{\int_{T-\tau_{2}}^{T} \int_{Q_{R}\left(x_{0}\right) \cap \Omega_{n}} \varphi_{n}^{2}(x, t) \mathrm{d} x \mathrm{~d} t}{\int_{B_{r}\left(x_{0}\right) \cap \Omega_{n}} \varphi_{n}^{2}(x, T) \mathrm{d} x}\right]}
$$

(where $\varphi_{n}$ satisfies the equation (2.1) with $\varphi_{0} \in L^{2}\left(\Omega_{n}\right) \backslash\{0\}$, and $\widetilde{C}_{1}>1$ is the constant given by Lem. A.1), has the following two properties:

(i)

$$
0<\left(1+4 \widetilde{C}_{3} T^{-1}+2 T\|a\|_{\infty}+\|a\|_{\infty}^{2 / 3}\right) h_{0}<\widetilde{C}_{3} .
$$

(ii) There is a constant $\widetilde{C}_{5} \triangleq \widetilde{C}_{5}(r, \delta)>\widetilde{C}_{3}$ so that

$$
e^{2 T\|a\|_{\infty}} \int_{T-\tau_{2}}^{T} \int_{Q_{R}\left(x_{0}\right) \cap \Omega_{n}} \varphi_{n}^{2}(x, s) \mathrm{d} x \mathrm{~d} s \leq e^{1+\frac{\tilde{C}_{5}}{h_{0}}} \int_{B_{(1+\delta) r}\left(x_{0}\right) \cap \Omega_{n}} \varphi_{n}^{2}(x, t) \mathrm{d} x
$$

for each $t \in\left[T-\min \left\{\tau_{2}, h_{0}\right\}, T\right]$.

Proof. For each $r^{\prime}>0$, we write $B_{r^{\prime}} \triangleq B_{r^{\prime}}\left(x_{0}\right)$ and $Q_{r^{\prime}} \triangleq Q_{r^{\prime}}\left(x_{0}\right)$. Since $B_{2 r} \subset Q_{R}$ and

$$
e^{2 \widetilde{C}_{1}\left(1+r^{-2}\right)\left[1+\left(\tau_{2}-\tau_{1}\right)^{-1}+\|a\|_{\infty}^{2 / 3}\right]} \geq \widetilde{C}_{1}\left[r^{-2}+\left(\tau_{2}-\tau_{1}\right)^{-1}+\|a\|_{\infty}\right],
$$

by (A.1) (where $R$ is replaced by $2 r$ ), we have

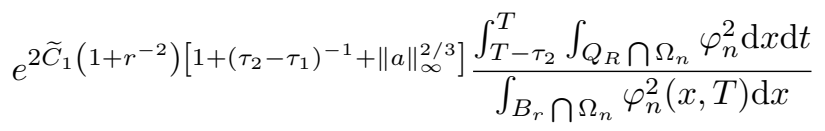




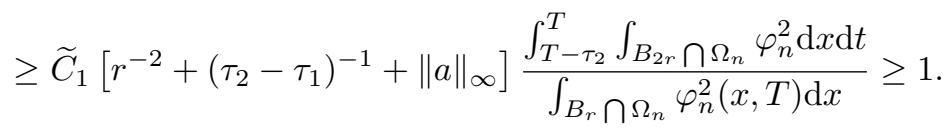

Hence, (A.16) follows immediately from (A.15).

We now turn to the proof of (A.17). Let $h>0, \beta(x)=\left|x-x_{0}\right|^{2}$ and $\eta \in C_{0}^{\infty}\left(B_{(1+\delta) r}\right)$ be such that

$$
0 \leq \eta(\cdot) \leq 1 \text { in } B_{(1+\delta) r} \text { and } \eta(\cdot)=1 \text { in } B_{(1+3 \delta / 4) r} .
$$

Multiplying the first equation of (2.1) by $e^{-\beta / h} \eta^{2} \varphi_{n}$ and integrating it over $B_{(1+\delta) r} \bigcap \Omega_{n}$, we get

$$
\begin{aligned}
& \frac{1}{2} \frac{\mathrm{d}}{\mathrm{d} t} \int_{B_{(1+\delta) r} \cap \Omega_{n}} e^{-\beta / h}\left(\eta \varphi_{n}\right)^{2} \mathrm{~d} x+\int_{B_{(1+\delta) r} \cap \Omega_{n}} \nabla \varphi_{n} \cdot \nabla\left(e^{-\beta / h} \eta^{2} \varphi_{n}\right) \mathrm{d} x \\
= & -\int_{B_{(1+\delta) r} \cap \Omega_{n}} a e^{-\beta / h}\left(\eta \varphi_{n}\right)^{2} \mathrm{~d} x .
\end{aligned}
$$

Since

$$
\nabla\left(e^{-\beta / h} \eta^{2} \varphi_{n}\right)=-\frac{1}{h} e^{-\beta / h} \eta^{2} \varphi_{n} \nabla \beta+2 e^{-\beta / h} \eta \varphi_{n} \nabla \eta+e^{-\beta / h} \eta^{2} \nabla \varphi_{n}
$$

by (A.18), we have

$$
\begin{aligned}
& \frac{1}{2} \frac{\mathrm{d}}{\mathrm{d} t} \int_{B_{(1+\delta) r} \cap \Omega_{n}} e^{-\beta / h}\left(\eta \varphi_{n}\right)^{2} \mathrm{~d} x+\int_{B_{(1+\delta) r} \cap \Omega_{n}} e^{-\beta / h}\left|\eta \nabla \varphi_{n}\right|^{2} \mathrm{~d} x \\
\leq & \int_{B_{(1+\delta) r} \cap \Omega_{n}} e^{-\beta /(2 h)}\left|\eta \nabla \varphi_{n}\right|\left(\frac{2}{h}\left|x-x_{0}\right| e^{-\beta /(2 h)} \eta\left|\varphi_{n}\right|+2|\nabla \eta| e^{-\beta /(2 h)}\left|\varphi_{n}\right|\right) \mathrm{d} x \\
& +\|a\|_{\infty} \int_{B_{(1+\delta) r} \cap \Omega_{n}} e^{-\beta / h}\left(\eta \varphi_{n}\right)^{2} \mathrm{~d} x .
\end{aligned}
$$

This, along with Cauchy-Schwarz inequality, implies that

$$
\begin{aligned}
\frac{\mathrm{d}}{\mathrm{d} t} \int_{B_{(1+\delta) r} \cap \Omega_{n}} e^{-\beta / h}\left(\eta \varphi_{n}\right)^{2} \mathrm{~d} x \leq & {\left[\frac{4(1+\delta)^{2} r^{2}}{h^{2}}+2\|a\|_{\infty}\right] \int_{B_{(1+\delta) r} \cap \Omega_{n}} e^{-\beta / h}\left(\eta \varphi_{n}\right)^{2} \mathrm{~d} x } \\
& +4 \int_{\left(B_{(1+\delta) r} \backslash B_{(1+3 \delta / 4) r}\right) \cap \Omega_{n}}|\nabla \eta|^{2} e^{-\beta / h} \varphi_{n}^{2} \mathrm{~d} x
\end{aligned}
$$

which indicates that

$$
\begin{aligned}
\frac{\mathrm{d}}{\mathrm{d} t} \int_{B_{(1+\delta) r} \cap \Omega_{n}} e^{-\beta / h}\left(\eta \varphi_{n}\right)^{2} \mathrm{~d} x \leq & {\left[\frac{4(1+\delta)^{2} r^{2}}{h^{2}}+2\|a\|_{\infty}\right] \int_{B_{(1+\delta) r} \cap \Omega_{n}} e^{-\beta / h}\left(\eta \varphi_{n}\right)^{2} \mathrm{~d} x } \\
& +4\|\nabla \eta\|_{\infty}^{2} e^{-\frac{(1+3 \delta / 4)^{2} r^{2}}{h}} \int_{B_{(1+\delta) r} \cap \Omega_{n}} \varphi_{n}^{2} \mathrm{~d} x
\end{aligned}
$$


Here and throughout the proof of Lemma A.3, $\|\nabla \eta\|_{\infty} \triangleq\|\nabla \eta\|_{L^{\infty}\left(B_{(1+\delta) r}\right)}$. From the latter it follows that

$$
\begin{aligned}
& \frac{\mathrm{d}}{\mathrm{d} t}\left[e^{-\left(\frac{4(1+\delta)^{2} r^{2}}{h^{2}}+2\|a\|_{\infty}\right) t} \int_{B_{(1+\delta) r} \cap \Omega_{n}} e^{-\beta / h}\left|\eta \varphi_{n}\right|^{2} \mathrm{~d} x\right] \\
\leq & 4\|\nabla \eta\|_{\infty}^{2} e^{-\left(\frac{4(1+\delta)^{2} r^{2}}{h^{2}}+2\|a\|_{\infty}\right) t} e^{-\frac{(1+3 \delta / 4)^{2} r^{2}}{h}} \int_{B_{(1+\delta) r} \cap \Omega_{n}} \varphi_{n}^{2} \mathrm{~d} x .
\end{aligned}
$$

Integrating the latter inequality over $(t, T)$, we get

$$
\begin{aligned}
& \int_{B_{(1+\delta) r} \cap \Omega_{n}} e^{-\beta / h}\left|\eta \varphi_{n}(x, T)\right|^{2} \mathrm{~d} x \\
\leq & e^{\left(\frac{4(1+\delta)^{2} r^{2}}{h^{2}}+2\|a\|_{\infty}\right)(T-t)} \int_{B_{(1+\delta) r} \cap \Omega_{n}} e^{-\beta / h}\left|\eta \varphi_{n}(x, t)\right|^{2} \mathrm{~d} x \\
& +4 e^{\left(\frac{4(1+\delta)^{2} r^{2}}{h^{2}}+2\|a\|_{\infty}\right)(T-t)}\|\nabla \eta\|_{\infty}^{2} e^{-\frac{(1+3 \delta / 4)^{2} r^{2}}{h}} \int_{t}^{T} \int_{B_{(1+\delta) r} \cap \Omega_{n}} \varphi_{n}^{2}(x, s) \mathrm{d} x \mathrm{~d} s .
\end{aligned}
$$

We simply write $b_{1} \triangleq 4(1+\delta)^{2}, b_{2} \triangleq(1+3 \delta / 4)^{2}$ and $b_{3} \triangleq(1+\delta / 2)^{2}$. It is clear that $1<b_{3}<b_{2}<b_{1}$. Recall that $t \leq T$. We now suppose $h>0$ to be such that

$$
0<T-\frac{\left(b_{2}-b_{3}\right) h}{b_{1}} \leq t
$$

Then $b_{1}(T-t) / h^{2} \leq\left(b_{2}-b_{3}\right) / h$ and (A.19) yields

$$
\begin{aligned}
& \int_{B_{(1+\delta) r} \cap \Omega_{n}} e^{-\beta / h}\left|\eta \varphi_{n}(x, T)\right|^{2} \mathrm{~d} x \leq e^{\frac{\left(b_{2}-b_{3}\right) r^{2}}{h}} e^{2 T\|a\|_{\infty}} \int_{B_{(1+\delta) r} \cap \Omega_{n}} e^{-\beta / h}\left|\eta \varphi_{n}(x, t)\right|^{2} \mathrm{~d} x \\
& +4\|\nabla \eta\|_{\infty}^{2} e^{2 T\|a\|_{\infty}} e^{\frac{-b_{3} r^{2}}{h}} \int_{t}^{T} \int_{B_{(1+\delta) r} \cap \Omega_{n}} \varphi_{n}^{2}(x, s) \mathrm{d} x \mathrm{~d} s .
\end{aligned}
$$

Since $\eta(\cdot)=1$ in $B_{r}$, the above estimate gives

$$
\begin{aligned}
\int_{B_{r} \cap \Omega_{n}}\left|\varphi_{n}(x, T)\right|^{2} \mathrm{~d} x \leq & e^{\frac{\left(b_{2}-b_{3}+1\right) r^{2}}{h}} e^{2 T\|a\|_{\infty}} \int_{B_{(1+\delta) r} \cap \Omega_{n}} e^{-\beta / h}\left|\eta \varphi_{n}(x, t)\right|^{2} \mathrm{~d} x \\
& +4\|\nabla \eta\|_{\infty}^{2} e^{2 T\|a\|_{\infty}} e^{\frac{-\left(b_{3}-1\right) r^{2}}{h}} \int_{t}^{T} \int_{B_{(1+\delta) r} \cap \Omega_{n}} \varphi_{n}^{2}(x, s) \mathrm{d} x \mathrm{~d} s
\end{aligned}
$$

whenever $0<T-\left(b_{2}-b_{3}\right) h / b_{1} \leq t \leq T$. Recall that $h_{0}<T$ from (A.16). We choose $h$ as follows:

$$
h=\frac{b_{1}}{b_{2}-b_{3}} h_{0}=\frac{b_{1} \widetilde{C}_{3} /\left(b_{2}-b_{3}\right)}{\ln \left[\left(1+\widetilde{C}_{4}\right)\left(e^{\left[1+2 \widetilde{C}_{1}\left(1+\frac{1}{r^{2}}\right)\right]\left(1+\frac{1}{\tau_{2}-\tau_{1}}+\|a\|_{\infty}^{2 / 3}\right)+\frac{4 \widetilde{C}_{3}}{T}+2 T\|a\|_{\infty}}\right) \frac{\int_{T-\tau_{2}}^{T} \int_{Q_{R}} \cap \Omega_{n} \varphi_{n}^{2} \mathrm{~d} x \mathrm{~d} t}{\int_{B_{r} \cap \Omega_{n}} \varphi_{n}^{2}(x, T) \mathrm{d} x}\right]}
$$


with $\widetilde{C}_{3} \triangleq\left(b_{2}-b_{3}\right)\left(b_{3}-1\right) r^{2} / b_{1}$ and $\widetilde{C}_{4} \triangleq 4\|\nabla \eta\|_{\infty}^{2}$. Then for any $t \in\left[T-\min \left\{\tau_{2}, h_{0}\right\}, T\right]$, we have

$$
\begin{aligned}
& 4\|\nabla \eta\|_{\infty}^{2} e^{2 T\|a\|_{\infty}} e^{-\frac{\left(b_{3}-1\right) r^{2}}{h}} \int_{t}^{T} \int_{B_{(1+\delta) r} \cap \Omega_{n}} \varphi_{n}^{2}(x, s) \mathrm{d} x \mathrm{~d} s \\
= & \frac{\widetilde{C}_{4} e^{2 T\|a\|_{\infty}} \int_{t}^{T} \int_{B_{(1+\delta) r} \cap \Omega_{n}} \varphi_{n}^{2}(x, s) \mathrm{d} x \mathrm{~d} s}{\left(1+\widetilde{C}_{4}\right)\left(e^{\left[1+2 \widetilde{C}_{1}\left(1+\frac{1}{r^{2}}\right)\right]\left(1+\frac{1}{\tau_{2}-\tau_{1}}+\|a\|_{\infty}^{2 / 3}\right)+\frac{4 \widetilde{C}_{3}}{T}+2 T\|a\|_{\infty}}\right) \frac{\int_{T-\tau_{2} \int_{Q_{R} \cap \Omega_{n}} \varphi_{n}^{2}(x, s) \mathrm{d} x \mathrm{~d} s}^{\int_{B_{r} \cap \Omega_{n}} \varphi_{n}^{2}(x, T) \mathrm{d} x}}{\leq}} \\
\leq & \frac{1}{e} \int_{B_{r} \cap \Omega_{n}} \varphi_{n}^{2}(x, T) \mathrm{d} x .
\end{aligned}
$$

(In the last inequality, we used the facts that $(1+\delta) r \leq 2 r \leq R$ and $B_{(1+\delta) r} \subset Q_{R}$.)

Next, on one hand, by (A.20) and (A.21), we get

$$
\left(1-\frac{1}{e}\right) \int_{B_{r} \cap \Omega_{n}} \varphi_{n}^{2}(x, T) \mathrm{d} x \leq e^{\frac{\left(b_{2}-b_{3}+1\right)\left(b_{2}-b_{3}\right) r^{2}}{b_{1} h_{0}}} e^{2 T\|a\|_{\infty}} \int_{B_{(1+\delta) r} \cap \Omega_{n}}\left|\varphi_{n}(x, t)\right|^{2} \mathrm{~d} x
$$

for each $T-\min \left\{\tau_{2}, h_{0}\right\} \leq t \leq T$. On the other hand, by (A.15), we see

$$
\frac{\int_{T-\tau_{2}}^{T} \int_{Q_{R} \cap \Omega_{n}} \varphi_{n}^{2}(x, s) \mathrm{d} x \mathrm{~d} s}{\int_{B_{r}} \cap \Omega_{n} \varphi_{n}^{2}(x, T) \mathrm{d} x} \leq e^{\frac{\widetilde{C}_{3}}{h_{0}}}
$$

which, combined with (A.22), indicates that

$$
\left(1-\frac{1}{e}\right) e^{-\frac{\tilde{C}_{3}}{h_{0}}} \int_{T-\tau_{2}}^{T} \int_{Q_{R} \cap \Omega_{n}} \varphi_{n}^{2}(x, s) \mathrm{d} x \mathrm{~d} s \leq e^{\frac{\left(b_{2}-b_{3}+1\right)\left(b_{2}-b_{3}\right) r^{2}}{b_{1} h_{0}}} e^{2 T\|a\|_{\infty}} \int_{B_{(1+\delta) r} \cap \Omega_{n}}\left|\varphi_{n}(x, t)\right|^{2} \mathrm{~d} x
$$

for each $T-\min \left\{\tau_{2}, h_{0}\right\} \leq t \leq T$. Since $2 T\|a\|_{\infty} h_{0}<\widetilde{C}_{3}$ (see (A.16)), the desired estimate (A.17) follows from the latter inequality immediately with $\widetilde{C}_{5} \triangleq 3 \widetilde{C}_{3}+\left(b_{2}-b_{3}+1\right)\left(b_{2}-b_{3}\right) r^{2} / b_{1}$.

Next, we introduce the following monotonicity of the generalized frequency function associated with parabolic equations in convex domains. It plays the exactly same role as that of Lemma 3.1 in [8].

Lemma A.4. ([19] or [27]) Let $r>0, \lambda>0, T>0$ and $x_{0} \in \Omega_{n}$. Denote

$$
G_{\lambda}(x, t) \triangleq \frac{1}{(T-t+\lambda)^{N / 2}} e^{-\frac{\left|x-x_{0}\right|^{2}}{4(T-t+\lambda)}}, \quad t \in[0, T]
$$

For $u \in H^{1}\left(0, T ; L^{2}\left(B_{r}\left(x_{0}\right) \bigcap \Omega_{n}\right)\right) \bigcap L^{2}\left(0, T ; H^{2}\left(B_{r}\left(x_{0}\right) \bigcap \Omega_{n}\right) \bigcap H_{0}^{1}\left(B_{r}\left(x_{0}\right) \bigcap \Omega_{n}\right)\right)$ and $t \in(0, T]$, set

$$
N_{\lambda, r}(t) \triangleq \frac{\int_{B_{r}\left(x_{0}\right) \cap \Omega_{n}}|\nabla u(x, t)|^{2} G_{\lambda}(x, t) \mathrm{d} x}{\int_{B_{r}\left(x_{0}\right) \cap \Omega_{n}}|u(x, t)|^{2} G_{\lambda}(x, t) \mathrm{d} x} \quad \text { whenever } \int_{B_{r}\left(x_{0}\right) \cap \Omega_{n}}|u(x, t)|^{2} \mathrm{~d} x \neq 0
$$

The following two properties hold: 
(i)

$$
\begin{aligned}
& \frac{1}{2} \frac{\mathrm{d}}{\mathrm{d} t} \int_{B_{r}\left(x_{0}\right) \cap \Omega_{n}}|u(x, t)|^{2} G_{\lambda}(x, t) \mathrm{d} x+\int_{B_{r}\left(x_{0}\right) \cap \Omega_{n}}|\nabla u(x, t)|^{2} G_{\lambda}(x, t) \mathrm{d} x \\
= & \int_{B_{r}\left(x_{0}\right) \cap \Omega_{n}} u(x, t)\left(\partial_{t}-\Delta\right) u(x, t) G_{\lambda}(x, t) \mathrm{d} x .
\end{aligned}
$$

(ii)

$$
\frac{\mathrm{d}}{\mathrm{d} t} N_{\lambda, r}(t) \leq \frac{1}{T-t+\lambda} N_{\lambda, r}(t)+\frac{\int_{B_{r}\left(x_{0}\right) \cap \Omega_{n}}\left|\left(\partial_{t} u-\Delta u\right)(x, t)\right|^{2} G_{\lambda}(x, t) \mathrm{d} x}{\int_{B_{r}\left(x_{0}\right) \cap \Omega_{n}}|u(x, t)|^{2} G_{\lambda}(x, t) \mathrm{d} x} .
$$

We are now in a position to present the proof of Proposition 2.5. Its proof below is almost the same as that of Lemma 3.2 in [8] by using Lemma A.4 instead.

Proof of Proposition 2.5. For each $r^{\prime}>0$, we denote $B_{r^{\prime}} \triangleq B_{r^{\prime}}\left(x_{0}\right)$ and $Q_{r^{\prime}} \triangleq Q_{r^{\prime}}\left(x_{0}\right)$. Let $\chi \in C_{0}^{\infty}\left(B_{R_{0}}\right)$ be such that

$$
0 \leq \chi(\cdot) \leq 1 \text { in } B_{R_{0}} \text { and } \chi(\cdot)=1 \text { in } B_{(1+3 \delta / 2) R} .
$$

We set $u \triangleq \chi \varphi_{n}$. It is clear that

$$
\partial_{t} u-\Delta u=-a u-2 \nabla \chi \cdot \nabla \varphi_{n}-\varphi_{n} \Delta \chi \text { in }\left(B_{R_{0}} \bigcap \Omega_{n}\right) \times(0, T)
$$

Furthermore, we define $g \triangleq-2 \nabla \chi \cdot \nabla \varphi_{n}-\varphi_{n} \Delta \chi$.

Step 1. Note that $g$ is supported on $\left\{x:(1+3 \delta / 2) R \leq\left|x-x_{0}\right| \leq R_{0}\right\}$. Recall that $\chi(\cdot)=1$ in $B_{(1+\delta) R}$. We can easily check that

$$
\begin{aligned}
& \frac{\int_{B_{R_{0}} \cap \Omega_{n}} u(x, t) g(x, t) G_{\lambda}(x, t) \mathrm{d} x}{\int_{B_{R_{0}} \cap \Omega_{n}}|u(x, t)|^{2} G_{\lambda}(x, t) \mathrm{d} x} \\
& =\frac{\int_{\left(B_{R_{0}} \backslash B_{(1+3 \delta / 2) R}\right) \cap \Omega_{n}} \chi \varphi_{n}\left(-2 \nabla \chi \cdot \nabla \varphi_{n}-\varphi_{n} \Delta \chi\right) e^{-\frac{\left|x-x_{0}\right|^{2}}{4(T-t+\lambda)}} \mathrm{d} x}{\int_{B_{R_{0}} \cap \Omega_{n}}\left|\chi \varphi_{n}(x, t)\right|^{2} e^{-\frac{\left|x-x_{0}\right|^{2}}{4(T-t+\lambda)}} \mathrm{d} x}
\end{aligned}
$$

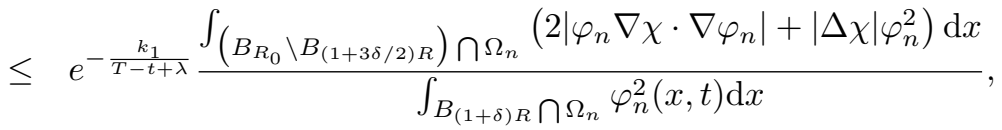

where $k_{1} \triangleq[(1+3 \delta / 2) R]^{2} / 4-[(1+\delta) R]^{2} / 4$. It follows from (A.25) that

$$
\frac{\int_{B_{R_{0}} \cap \Omega_{n}} u(x, t) g(x, t) G_{\lambda}(x, t) \mathrm{d} x}{\int_{B_{R_{0}} \cap \Omega_{n}}|u(x, t)|^{2} G_{\lambda}(x, t) \mathrm{d} x}
$$




$$
\leq e^{-\frac{k_{1}}{T-t+\lambda}} \frac{2\|\nabla \chi\|_{\infty}\left(\int_{B_{R_{0}}} \cap \Omega_{n} \varphi_{n}^{2}(x, t) \mathrm{d} x\right)^{\frac{1}{2}}\left(\int_{B_{R_{0}} \cap \Omega_{n}}\left|\nabla \varphi_{n}(x, t)\right|^{2} \mathrm{~d} x\right)^{\frac{1}{2}}+\|\Delta \chi\|_{\infty} \int_{B_{R_{0}} \cap \Omega_{n}} \varphi_{n}^{2}(x, t) \mathrm{d} x}{\int_{B_{(1+\delta) R}} \cap \Omega_{n} \varphi_{n}^{2}(x, t) \mathrm{d} x} .
$$

Here $\|\nabla \chi\|_{\infty} \triangleq\|\nabla \chi\|_{L^{\infty}\left(B_{R_{0}}\right)}$ and $\|\Delta \chi\|_{\infty} \triangleq\|\Delta \chi\|_{L^{\infty}\left(B_{R_{0}}\right)}$.

On one hand, by Lemma A.1 (where $r, R, \tau_{1}$ and $\tau_{2}$ are replaced by $R_{0}, 2 R_{0}, T / 4$ and $T / 2$, respectively), we have

$$
\int_{B_{R_{0}} \cap \Omega_{n}} \varphi_{n}^{2}(x, t) \mathrm{d} x \leq k_{2}\left(1+T^{-1}+\|a\|_{\infty}\right) \int_{T / 2}^{T} \int_{B_{2 R_{0}} \cap \Omega_{n}} \varphi_{n}^{2}(x, s) \mathrm{d} x \mathrm{~d} s \text { for each } t \in[3 T / 4, T],
$$

where $k_{2} \triangleq k_{2}(R)>0$. By Lemma A.2 (where $R$ and $\tau$ are replaced by $R_{0}$ and $T / 4$, respectively), we get

$$
\begin{aligned}
& \int_{B_{R_{0}} \cap \Omega_{n}}\left|\nabla \varphi_{n}(x, t)\right|^{2} \mathrm{~d} x \\
\leq & k_{3}\left(1+T^{-2}+\|a\|_{\infty}^{2}\right) \int_{T / 2}^{T} \int_{B_{2 R_{0}} \cap \Omega_{n}} \varphi_{n}^{2}(x, s) \mathrm{d} x \mathrm{~d} s \text { for each } t \in[3 T / 4, T],
\end{aligned}
$$

where $k_{3} \triangleq k_{3}(R)>0$. It follows from (A.26)-(A.28) that

$$
\begin{aligned}
& \frac{\int_{B_{R_{0}} \cap \Omega_{n}} u(x, t) g(x, t) G_{\lambda}(x, t) \mathrm{d} x}{\int_{B_{R_{0}} \cap \Omega_{n}}|u(x, t)|^{2} G_{\lambda}(x, t) \mathrm{d} x} \\
\leq & e^{-\frac{k_{1}}{T-t+\lambda}} \frac{k_{4}\left(1+T^{-2}+\|a\|_{\infty}^{3 / 2}\right) \int_{T / 2}^{T} \int_{B_{2 R_{0}} \cap \Omega_{n}} \varphi_{n}^{2}(x, s) \mathrm{d} x \mathrm{~d} s}{\int_{B_{(1+\delta) R} \cap \Omega_{n}} \varphi_{n}^{2}(x, t) \mathrm{d} x} \text { for each } t \in[3 T / 4, T],
\end{aligned}
$$

where $k_{4} \triangleq k_{4}(R, \delta)>0$.

According to (A.17) (where $r, R, \tau_{1}$ and $\tau_{2}$ are replaced by $R, 2 R_{0}, T / 4$ and $T / 2$, respectively), it holds that

$$
\begin{aligned}
e^{2 T\|a\|_{\infty}} \int_{T / 2}^{T} \int_{B_{2 R_{0}} \cap \Omega_{n}} \varphi_{n}^{2} \mathrm{~d} x \mathrm{~d} s & \leq e^{2 T\|a\|_{\infty}} \int_{T / 2}^{T} \int_{Q_{2 R_{0}} \cap \Omega_{n}} \varphi_{n}^{2} \mathrm{~d} x \mathrm{~d} s \\
& \leq e^{1+\frac{\widetilde{\sigma}_{5}}{h_{0}}} \int_{B_{(1+\delta) R} \cap \Omega_{n}} \varphi_{n}^{2}(x, t) \mathrm{d} x \text { for each } t \in\left[T-h_{0}, T\right] .
\end{aligned}
$$

Here, we used the fact that $h_{0}<T / 4$ (see (A.16)). This, along with (A.29), implies that

$$
\frac{\int_{B_{R_{0}} \cap \Omega_{n}} u(x, t) g(x, t) G_{\lambda}(x, t) \mathrm{d} x}{\int_{B_{R_{0}} \cap \Omega_{n}}|u(x, t)|^{2} G_{\lambda}(x, t) \mathrm{d} x} \leq k_{4} e^{-\frac{k_{1}}{T-t+\lambda}} e^{1+\frac{\tilde{C}_{5}}{h_{0}}}\left(1+T^{-2}\right) \quad \text { for each } t \in\left[T-h_{0}, T\right] .
$$

On the other hand, by similar arguments as those for (A.26), we have

$$
\int_{t}^{T} \frac{\int_{B_{R_{0}} \cap \Omega_{n}}|g(x, s)|^{2} G_{\lambda}(x, s) \mathrm{d} x}{\int_{B_{R_{0}} \cap \Omega_{n}}|u(x, s)|^{2} G_{\lambda}(x, s) \mathrm{d} x} \mathrm{~d} s
$$




$$
\begin{aligned}
& \leq \int_{t}^{T} \frac{\int_{B_{R_{0}} \cap \Omega_{n}}\left|-2 \nabla \chi \cdot \nabla \varphi_{n}-\varphi_{n} \Delta \chi\right|^{2} \mathrm{~d} x}{\int_{B_{(1+\delta) R}} \cap \Omega_{n}\left|\varphi_{n}(x, s)\right|^{2} \mathrm{~d} x} e^{-\frac{k_{1}}{T-s+\lambda}} \mathrm{d} s \\
& \leq \int_{t}^{T} \frac{8\|\nabla \chi\|_{\infty}^{2} \int_{B_{R_{0}} \cap \Omega_{n}}\left|\nabla \varphi_{n}\right|^{2} \mathrm{~d} x+2\|\Delta \chi\|_{\infty}^{2} \int_{B_{R_{0}} \cap \Omega_{n}} \varphi_{n}^{2} \mathrm{~d} x}{\int_{B_{(1+\delta) R} \cap \Omega_{n}}\left|\varphi_{n}(x, s)\right|^{2} \mathrm{~d} x} e^{-\frac{k_{1}}{T-s+\lambda}} \mathrm{d} s
\end{aligned}
$$

for each $t \in[3 T / 4, T]$. This, together with (A.27) and (A.28), yields

$$
\begin{aligned}
& \int_{t}^{T} \frac{\int_{B_{R_{0}} \cap \Omega_{n}}|g(x, s)|^{2} G_{\lambda}(x, s) \mathrm{d} x}{\int_{B_{R_{0}} \cap \Omega_{n}}|u(x, s)|^{2} G_{\lambda}(x, s) \mathrm{d} x} \mathrm{~d} s \\
\leq & k_{5}\left(1+T^{-2}+\|a\|_{\infty}^{2}\right) \int_{t}^{T} \frac{\int_{T / 2}^{T} \int_{B_{2 R_{0}} \cap \Omega_{n}} \varphi_{n}^{2} \mathrm{~d} x \mathrm{~d} s}{\int_{B_{(1+\delta) R} \cap \Omega_{n}}\left|\varphi_{n}(x, s)\right|^{2} \mathrm{~d} x} e^{-\frac{k_{1}}{T-s+\lambda}} \mathrm{d} s
\end{aligned}
$$

for each $t \in[3 T / 4, T]$, where $k_{5} \triangleq k_{5}(R, \delta)>0$. It follows from (A.30) and (A.32) that

$$
\begin{aligned}
& \int_{t}^{T} \frac{\int_{B_{R_{0}} \cap \Omega_{n}}|g(x, s)|^{2} G_{\lambda}(x, s) \mathrm{d} x}{\int_{B_{R_{0}} \cap \Omega_{n}}|u(x, s)|^{2} G_{\lambda}(x, s) \mathrm{d} x} \mathrm{~d} s \\
& \leq k_{5}\left(1+T^{-2}+\|a\|_{\infty}^{2}\right) e^{1+\frac{\widetilde{C}_{5}}{h_{0}}} e^{-2 T\|a\|_{\infty}} \int_{t}^{T} e^{-\frac{k_{1}}{T-s+\lambda}} \mathrm{d} s \\
& \leq k_{5}\left(1+T^{-2}\right) e^{1+\frac{\widetilde{C}_{5}}{h_{0}}} e^{-\frac{k_{1}}{T-t+\lambda}}(T-t) \text { for each } t \in\left[T-h_{0}, T\right] .
\end{aligned}
$$

Step 2. In this step, the aim is to give an upper bound for the term $\lambda N_{\lambda, R_{0}}(T)$ (i.e., (A.42) below). By (ii) of Lemma A.4 and (A.24), we get

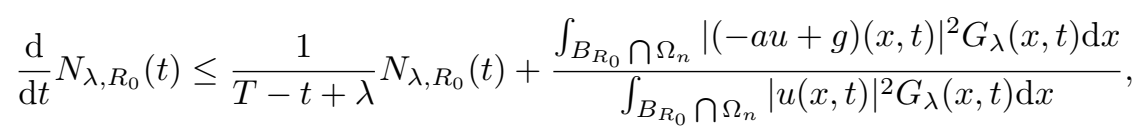

which indicates that

$$
\begin{aligned}
\frac{\mathrm{d}}{\mathrm{d} t}\left[(T-t+\lambda) N_{\lambda, R_{0}}(t)\right] & \leq(T-t+\lambda) \frac{\int_{B_{R_{0}} \cap \Omega_{n}}|(-a u+g)(x, t)|^{2} G_{\lambda}(x, t) \mathrm{d} x}{\int_{B_{R_{0}}} \cap \Omega_{n}|u(x, t)|^{2} G_{\lambda}(x, t) \mathrm{d} x} \\
& \leq 2(T-t+\lambda)\left(\|a\|_{\infty}^{2}+\frac{\int_{B_{R_{0}} \cap \Omega_{n}}|g(x, t)|^{2} G_{\lambda}(x, t) \mathrm{d} x}{\int_{B_{R_{0}} \cap \Omega_{n}}|u(x, t)|^{2} G_{\lambda}(x, t) \mathrm{d} x}\right) .
\end{aligned}
$$

Integrating the latter inequality over $(t, T)$, we obtain

$$
\begin{aligned}
\lambda N_{\lambda, R_{0}}(T) \leq & (T-t+\lambda) N_{\lambda, R_{0}}(t)+2\|a\|_{\infty}^{2} \int_{t}^{T}(T-s+\lambda) \mathrm{d} s \\
& +2 \int_{t}^{T}(T-s+\lambda) \frac{\int_{B_{R_{0}} \cap \Omega_{n}}|g(x, s)|^{2} G_{\lambda}(x, s) \mathrm{d} x}{\int_{B_{R_{0}} \cap \Omega_{n}}|u(x, s)|^{2} G_{\lambda}(x, s) \mathrm{d} x} \mathrm{~d} s .
\end{aligned}
$$


Hence, for any $0<T-\varepsilon \leq t<T$ (where $\varepsilon$ will be determined later), we have

$$
\frac{\lambda}{\varepsilon+\lambda} N_{\lambda, R_{0}}(T) \leq N_{\lambda, R_{0}}(t)+2 \varepsilon\|a\|_{\infty}^{2}+2 \int_{t}^{T} \frac{\int_{B_{R_{0}} \cap \Omega_{n}}|g(x, s)|^{2} G_{\lambda}(x, s) \mathrm{d} x}{\int_{B_{R_{0}} \cap \Omega_{n}}|u(x, s)|^{2} G_{\lambda}(x, s) \mathrm{d} x} \mathrm{~d} s .
$$

Moreover, by (i) of Lemma A.4 and (A.24), we observe that

$$
\begin{aligned}
& \frac{1}{2} \frac{\mathrm{d}}{\mathrm{d} t} \int_{B_{R_{0}} \cap \Omega_{n}}|u(x, t)|^{2} G_{\lambda}(x, t) \mathrm{d} x+N_{\lambda, R_{0}}(t) \int_{B_{R_{0}} \cap \Omega_{n}}|u(x, t)|^{2} G_{\lambda}(x, t) \mathrm{d} x \\
= & -\int_{B_{R_{0}} \cap \Omega_{n}} a(x, t)|u(x, t)|^{2} G_{\lambda}(x, t) \mathrm{d} x \\
& +\frac{\int_{B_{R_{0}} \cap \Omega_{n}} u(x, t) g(x, t) G_{\lambda}(x, t) \mathrm{d} x}{\int_{B_{R_{0}} \cap \Omega_{n}}|u(x, t)|^{2} G_{\lambda}(x, t) \mathrm{d} x} \int_{B_{R_{0}} \cap \Omega_{n}}|u(x, t)|^{2} G_{\lambda}(x, t) \mathrm{d} x .
\end{aligned}
$$

This, along with (A.34), implies

$$
\begin{aligned}
& \frac{1}{2} \frac{\mathrm{d}}{\mathrm{d} t} \int_{B_{R_{0}} \cap \Omega_{n}}|u(x, t)|^{2} G_{\lambda}(x, t) \mathrm{d} x+\frac{\lambda}{\varepsilon+\lambda} N_{\lambda, R_{0}}(T) \int_{B_{R_{0}} \cap \Omega_{n}}|u(x, t)|^{2} G_{\lambda}(x, t) \mathrm{d} x \\
\leq & \left(\|a\|_{\infty}+2 \varepsilon\|a\|_{\infty}^{2}\right) \int_{B_{R_{0}} \cap \Omega_{n}}|u(x, t)|^{2} G_{\lambda}(x, t) \mathrm{d} x+\int_{B_{R_{0}} \cap \Omega_{n}}|u(x, t)|^{2} G_{\lambda}(x, t) \mathrm{d} x \\
& \times\left(\frac{\int_{B_{R_{0}} \cap \Omega_{n}} u(x, t) g(x, t) G_{\lambda}(x, t) \mathrm{d} x}{\int_{B_{R_{0}} \cap \Omega_{n}}|u(x, t)|^{2} G_{\lambda}(x, t) \mathrm{d} x}+2 \int_{t}^{T} \frac{\int_{B_{R_{0}} \cap \Omega_{n}}|g(x, s)|^{2} G_{\lambda}(x, s) \mathrm{d} x}{\int_{B_{R_{0}} \cap \Omega_{n}}|u(x, s)|^{2} G_{\lambda}(x, s) \mathrm{d} x} \mathrm{~d} s\right) .
\end{aligned}
$$

Next, on one hand, it follows from (A.31) and (A.33) that

$$
\begin{aligned}
& \frac{\int_{B_{R_{0}} \cap \Omega_{n}} u(x, t) g(x, t) G_{\lambda}(x, t) \mathrm{d} x}{\int_{B_{R_{0}} \cap \Omega_{n}}|u(x, t)|^{2} G_{\lambda}(x, t) \mathrm{d} x}+2 \int_{t}^{T} \frac{\int_{B_{R_{0}} \cap \Omega_{n}}|g(x, s)|^{2} G_{\lambda}(x, s) \mathrm{d} x}{\int_{B_{R_{0}} \cap \Omega_{n}}|u(x, s)|^{2} G_{\lambda}(x, s) \mathrm{d} x} \mathrm{~d} s \\
\leq & k_{6}(1+\varepsilon)\left(1+T^{-2}\right) e^{-\frac{k_{1}}{\varepsilon+\lambda}} e^{\frac{\widetilde{C}_{5}+k_{1}}{h_{0}}} \\
\triangleq & Q_{h_{0}, \varepsilon, \lambda} \text { for each } 0<T-\varepsilon \leq t<T \text { with } \varepsilon \in\left(0, h_{0}\right],
\end{aligned}
$$

where $k_{6} \triangleq k_{6}(R, \delta)>0$. This, along with (A.35), implies that

$$
\begin{aligned}
& \frac{1}{2} \frac{\mathrm{d}}{\mathrm{d} t} \int_{B_{R_{0}} \cap \Omega_{n}}|u(x, t)|^{2} G_{\lambda}(x, t) \mathrm{d} x \\
\leq & -\left(\frac{\lambda}{\varepsilon+\lambda} N_{\lambda, R_{0}}(T)-\|a\|_{\infty}-2 \varepsilon\|a\|_{\infty}^{2}-Q_{h_{0}, \varepsilon, \lambda}\right) \int_{B_{R_{0}} \cap \Omega_{n}}|u(x, t)|^{2} G_{\lambda}(x, t) \mathrm{d} x,
\end{aligned}
$$

which indicates that

$$
\frac{\mathrm{d}}{\mathrm{d} t}\left[e^{2\left(\frac{\lambda}{\varepsilon+\lambda} N_{\lambda, R_{0}}(T)-\|a\|_{\infty}-2 \varepsilon\|a\|_{\infty}^{2}-Q_{h_{0}, \varepsilon, \lambda}\right) t} \int_{B_{R_{0}} \cap \Omega_{n}}|u(x, t)|^{2} G_{\lambda}(x, t) \mathrm{d} x\right] \leq 0
$$


for each $0<T-\varepsilon \leq t<T$ with $\varepsilon \in\left(0, h_{0}\right]$. Integrating the latter inequality over $(T-\varepsilon, T-\varepsilon / 2)$, we obtain

$$
\begin{aligned}
& e^{\frac{\varepsilon \lambda}{\varepsilon+\lambda} N_{\lambda, R_{0}}(T)} \int_{B_{R_{0}} \cap \Omega_{n}}|u(x, T-\varepsilon / 2)|^{2} G_{\lambda}(x, T-\varepsilon / 2) \mathrm{d} x \\
\leq & e^{\varepsilon\|a\|_{\infty}+2 \varepsilon^{2}\|a\|_{\infty}^{2}+\varepsilon Q_{h_{0}, \varepsilon, \lambda}} \int_{B_{R_{0}} \cap \Omega_{n}}|u(x, T-\varepsilon)|^{2} G_{\lambda}(x, T-\varepsilon) \mathrm{d} x .
\end{aligned}
$$

This yields

$$
e^{\frac{\varepsilon \lambda}{\varepsilon+\lambda} N_{\lambda, R_{0}}(T)} \leq e^{\varepsilon\|a\|_{\infty}+2 \varepsilon^{2}\|a\|_{\infty}^{2}+\varepsilon Q_{h_{0}, \varepsilon, \lambda}} \frac{\int_{B_{R_{0}} \cap \Omega_{n}}|u(x, T-\varepsilon)|^{2} e^{-\frac{\left|x-x_{0}\right|^{2}}{4(\varepsilon+\lambda)}} \mathrm{d} x}{\int_{B_{R_{0}} \cap \Omega_{n}}|u(x, T-\varepsilon / 2)|^{2} e^{-\frac{\left|x-x_{0}\right|^{2}}{4(\varepsilon / 2+\lambda)}} \mathrm{d} x} .
$$

On the other hand, by (A.27), we see

$$
\begin{aligned}
\frac{\int_{B_{R_{0}} \cap \Omega_{n}}|u(x, T-\varepsilon)|^{2} e^{-\frac{\left|x-x_{0}\right|^{2}}{4(\varepsilon+\lambda)}} \mathrm{d} x}{\int_{B_{R_{0}} \cap \Omega_{n}}|u(x, T-\varepsilon / 2)|^{2} e^{-\frac{\left|x-x_{0}\right|^{2}}{4(\varepsilon / 2+\lambda)} \mathrm{d} x}} & \leq \frac{e^{\frac{((1+\delta) R)^{2}}{4(\varepsilon / 2+\lambda)}} \int_{B_{R_{0}} \cap \Omega_{n}}\left|\varphi_{n}(x, T-\varepsilon)\right|^{2} \mathrm{~d} x}{\int_{B_{(1+\delta) R} \cap \Omega_{n}}\left|\varphi_{n}(x, T-\varepsilon / 2)\right|^{2} \mathrm{~d} x} \\
& \leq \frac{e^{\frac{((1+\delta) R)^{2}}{2 \varepsilon}} k_{2}\left(1+T^{-1}+\|a\|_{\infty}\right) \int_{T / 2}^{T} \int_{Q_{2 R_{0}} \cap \Omega_{n}} \varphi_{n}^{2} \mathrm{~d} x \mathrm{~d} t}{\int_{B_{(1+\delta) R} \cap \Omega_{n}}\left|\varphi_{n}(x, T-\varepsilon / 2)\right|^{2} \mathrm{~d} x},
\end{aligned}
$$

which, combined with (ii) of Lemma A.3 (where $r, R, \tau_{1}$ and $\tau_{2}$ are replaced by $R, 2 R_{0}, T / 4$ and $T / 2$, respectively), indicates that

$$
\begin{aligned}
\frac{\int_{B_{R_{0}} \cap \Omega_{n}}|u(x, T-\varepsilon)|^{2} e^{-\frac{\left|x-x_{0}\right|^{2}}{4(\varepsilon+\lambda)}} \mathrm{d} x}{\int_{B_{R_{0}} \cap \Omega_{n}}|u(x, T-\varepsilon / 2)|^{2} e^{-\frac{\left|x-x_{0}\right|^{2}}{4(\varepsilon / 2+\lambda)} \mathrm{d} x}} & \leq \frac{e^{\frac{((1+\delta) R)^{2}}{2 \varepsilon}} k_{2}\left(1+T^{-1}+\|a\|_{\infty}\right) e^{1+\frac{\widetilde{C}_{5}}{h_{0}}}}{e^{2 T\|a\|_{\infty}}} \\
& \leq k_{2} e^{\frac{((1+\delta) R)^{2}}{2 \varepsilon}}\left(1+T^{-1}\right) e^{1+\frac{\widetilde{C}_{5}}{h_{0}}} .
\end{aligned}
$$

Then, it follows from (A.37) and (A.38) that for each $\varepsilon \in\left(0, h_{0}\right]$,

$$
\begin{aligned}
& \lambda N_{\lambda, R_{0}}(T) \\
\leq & \frac{\varepsilon+\lambda}{\varepsilon}\left[\varepsilon\|a\|_{\infty}+2 \varepsilon^{2}\|a\|_{\infty}^{2}+\varepsilon Q_{h_{0}, \varepsilon, \lambda}+\frac{(1+\delta)^{2} R^{2}}{2 \varepsilon}+1+\frac{\widetilde{C}_{5}}{h_{0}}+\ln \left(k_{2}\left(1+T^{-1}\right)\right)\right] .
\end{aligned}
$$

Finally, we choose $\lambda=\mu \varepsilon$ with $\mu \in(0,1)$ (which will be determined later) and $\varepsilon=k_{1} h_{0} /\left[2\left(\widetilde{C}_{5}+k_{1}\right)\right]$ so that $Q_{h_{0}, \varepsilon, \lambda}($ see $(\mathrm{A} .36))$ satisfies

$$
\begin{aligned}
Q_{h_{0}, \varepsilon, \lambda} & =k_{6}(1+\varepsilon)\left(1+T^{-2}\right) e^{\frac{\widetilde{C}_{5}+k_{1}}{h_{0}}\left(\frac{\mu-1}{\mu+1}\right)} \\
& \leq k_{6}(1+\varepsilon)\left(1+T^{-2}\right) .
\end{aligned}
$$


Since $\varepsilon \leq h_{0}$, by (A.39) and (A.40), we get

$$
\begin{aligned}
\lambda N_{\lambda, R_{0}}(T) \leq & 2\left[h_{0}\|a\|_{\infty}+2 h_{0}^{2}\|a\|_{\infty}^{2}+k_{6}(1+\varepsilon)\left(1+T^{-2}\right) h_{0}\right] \\
& +2\left[1+\frac{\widetilde{C}_{5}}{h_{0}}+k_{2}\left(1+T^{-1}\right)+\frac{\widetilde{C}_{5}+k_{1}}{k_{1} h_{0}}(1+\delta)^{2} R^{2}\right] .
\end{aligned}
$$

According to $(i)$ of Lemma A.3 (where $r, R, \tau_{1}$ and $\tau_{2}$ are replaced by $R, 2 R_{0}, T / 4$ and $T / 2$, respectively), it is clear that

$$
h_{0}<\widetilde{C}_{3}, h_{0}<T, h_{0} T\|a\|_{\infty}<\widetilde{C}_{3} \text { and } h_{0}^{3}\|a\|_{\infty}^{2}<\widetilde{C}_{3}^{3} .
$$

These, together with (A.41), derive that

$$
\begin{aligned}
\varepsilon \lambda N_{\lambda, R_{0}}(T) \leq & 2\left[h_{0} T\|a\|_{\infty}+2 h_{0}^{3}\|a\|_{\infty}^{2}+k_{6}\left(1+h_{0}\right)\left(h_{0}^{2}+h_{0}^{2} T^{-2}\right)\right] \\
& +2\left[h_{0}+\widetilde{C}_{5}+k_{2} h_{0}\left(1+T^{-1}\right)+\frac{\widetilde{C}_{5}+k_{1}}{k_{1}}(1+\delta)^{2} R^{2}\right] \\
\leq & 2\left[\widetilde{C}_{3}+2 \widetilde{C}_{3}^{3}+k_{6}\left(1+\widetilde{C}_{3}\right)\left(1+\widetilde{C}_{3}^{2}\right)\right] \\
& +2\left[\widetilde{C}_{3}+\widetilde{C}_{5}+k_{2}\left(1+\widetilde{C}_{3}\right)+\frac{\widetilde{C}_{5}+k_{1}}{k_{1}}(1+\delta)^{2} R^{2}\right] .
\end{aligned}
$$

Hence,

$$
\frac{16 \lambda}{r^{2}}\left(\frac{N}{4}+\lambda N_{\lambda, R_{0}}(T)\right) \leq \frac{16 \mu}{r^{2}}\left(\frac{N}{4} \widetilde{C}_{3}+\varepsilon \lambda N_{\lambda, R_{0}}(T)\right) \leq \mu\left(1+k_{7}\right),
$$

where $k_{7} \triangleq k_{7}(r, R, \delta)>0$.

Step 3. We claim that

$$
\begin{aligned}
\int_{B_{R_{0}} \cap \Omega_{n}}|u(x, T)|^{2} e^{-\frac{\left|x-x_{0}\right|^{2}}{4 \lambda}} \mathrm{d} x \leq & \int_{B_{r} \cap \Omega_{n}}\left|\varphi_{n}(x, T)\right|^{2} e^{-\frac{\left|x-x_{0}\right|^{2}}{4 \lambda}} \mathrm{d} x \\
& +\mu\left(1+k_{7}\right) \int_{B_{R_{0}} \cap \Omega_{n}}|u(x, T)|^{2} e^{-\frac{\left|x-x_{0}\right|^{2}}{4 \lambda}} \mathrm{d} x .
\end{aligned}
$$

Indeed, since $B_{R_{0}} \bigcap \Omega_{n}$ is convex, we have (see, for instance, [19])

$$
\begin{aligned}
& \frac{1}{16 \lambda} \int_{B_{R_{0}} \cap \Omega_{n}}\left|x-x_{0}\right|^{2}|u(x, T)|^{2} e^{-\frac{\left|x-x_{0}\right|^{2}}{4 \lambda}} \mathrm{d} x \\
\leq & \frac{N}{4} \int_{B_{R_{0}} \cap \Omega_{n}}|u(x, T)|^{2} e^{-\frac{\left|x-x_{0}\right|^{2}}{4 \lambda}} \mathrm{d} x+\lambda \int_{B_{R_{0}} \cap \Omega_{n}}|\nabla u(x, T)|^{2} e^{-\frac{\left|x-x_{0}\right|^{2}}{4 \lambda}} \mathrm{d} x .
\end{aligned}
$$

This implies

$$
\int_{B_{R_{0}} \cap \Omega_{n}}|u(x, T)|^{2} e^{-\frac{\left|x-x_{0}\right|^{2}}{4 \lambda}} \mathrm{d} x
$$




$$
\begin{aligned}
& \leq \int_{\left(B_{R_{0}} \backslash B_{r}\right) \cap \Omega_{n}} \frac{\left|x-x_{0}\right|^{2}}{r^{2}}|u(x, T)|^{2} e^{-\frac{\left|x-x_{0}\right|^{2}}{4 \lambda}} \mathrm{d} x+\int_{B_{r} \cap \Omega_{n}}|u(x, T)|^{2} e^{-\frac{\left|x-x_{0}\right|^{2}}{4 \lambda}} \mathrm{d} x \\
& \leq \frac{16 \lambda}{r^{2}}\left[\frac{N}{4}+\lambda N_{\lambda, R_{0}}(T)\right] \int_{B_{R_{0}} \cap \Omega_{n}}|u(x, T)|^{2} e^{-\frac{\left|x-x_{0}\right|^{2}}{4 \lambda}} \mathrm{d} x+\int_{B_{r} \cap \Omega_{n}}\left|\varphi_{n}(x, T)\right|^{2} e^{-\frac{\left|x-x_{0}\right|^{2}}{4 \lambda}} \mathrm{d} x,
\end{aligned}
$$

where in the last line, we used the definition of $N_{\lambda, R_{0}}(T)$ and the fact that $u=\varphi_{n}$ in $B_{r} \bigcap \Omega_{n}$. Then (A.43) follows from (A.44) and (A.42) immediately.

Step 4. End of the proof.

We choose $\mu=1 /\left[2\left(1+k_{7}\right)\right]$. Then, $\lambda=\mu \varepsilon=k_{1} h_{0} /\left[4\left(1+k_{7}\right)\left(\widetilde{C}_{5}+k_{1}\right)\right]$. By (A.43), we have

$$
\begin{aligned}
\int_{B_{R} \cap \Omega_{n}}\left|\varphi_{n}(x, T)\right|^{2} d x & \leq e^{\frac{R^{2}}{4 \lambda}} \int_{B_{R_{0}} \cap \Omega_{n}}|u(x, T)|^{2} e^{-\frac{\left|x-x_{0}\right|^{2}}{4 \lambda}} \mathrm{d} x \\
& \leq 2 e^{\frac{R^{2}}{4 \lambda}} \int_{B_{r} \cap \Omega_{n}}\left|\varphi_{n}(x, T)\right|^{2} e^{-\frac{\left|x-x_{0}\right|^{2}}{4 \lambda}} \mathrm{d} x .
\end{aligned}
$$

This, along with the definition of $h_{0}$ (see (A.15), where $r, R, \tau_{1}$ and $\tau_{2}$ are replaced by $R, 2 R_{0}, T / 4$ and $T / 2$, respectively), implies that

$$
\begin{aligned}
& \int_{B_{R} \cap \Omega_{n}}\left|\varphi_{n}(x, T)\right|^{2} d x \leq 2 e^{\frac{\left(1+k_{7}\right)\left(\widetilde{C}_{5}+k_{1}\right) R^{2}}{k_{1} h_{0}}} \int_{B_{r} \cap \Omega_{n}}\left|\varphi_{n}(x, T)\right|^{2} \mathrm{~d} x \\
\leq & {\left[\left(1+\widetilde{C}_{4}\right)\left(e^{\left[1+2 \widetilde{C}_{1}\left(1+R^{-2}\right)\right]\left(1+4 T^{-1}+\|a\|_{\infty}^{2 / 3}\right)+\frac{4 \widetilde{C}_{3}}{T}+2 T\|a\|_{\infty}}\right) \frac{\int_{T / 2}^{T} \int_{Q_{2 R_{0}} \cap \Omega_{n}} \varphi_{n}^{2} \mathrm{~d} x \mathrm{~d} t}{\int_{B_{R} \cap \Omega_{n}}\left|\varphi_{n}(x, T)\right|^{2} \mathrm{~d} x}\right] } \\
& \times 2 \int_{B_{r} \cap \Omega_{n}}\left|\varphi_{n}(x, T)\right|^{2} \mathrm{~d} x .
\end{aligned}
$$

Hence, we can conclude that the desired estimate of Proposition 2.5 holds with

$$
\gamma=\frac{\left(1+k_{7}\right)\left(\widetilde{C}_{5}+k_{1}\right) R^{2}}{\widetilde{C}_{3} k_{1}+\left(1+k_{7}\right)\left(\widetilde{C}_{5}+k_{1}\right) R^{2}} \in(0,1) .
$$

In summary, we finish the proof of this proposition.

Acknowledgements. This work was partially supported by the National Natural Science Foundation of China under grants 12171377,11771344 and 11971363 . The second author is also partially supported by the Academic Team Building Plan for Young Scholars from Wuhan University under grant 413100085.

\section{REFERENCES}

[1] J. Apraiz, L. Escauriaza, G. Wang and C. Zhang, Observability inequalities and measurable sets. J. Eur. Math. Soc. 16 (2014) 2433-2475.

[2] V. Barbu, Exact controllability of the superlinear heat equation. Appl. Math. Optim. 42 (2000) 73-89.

[3] V. Barbu, Exact null internal controllability for the heat equation on unbounded convex domains. ESAIM: COCV 20 (2014) $222-235$.

[4] V.R. Cabanillas, S.B. de Menezes and E. Zuazua, Null controllability in unbounded domains for the semilinear heat equation with nonlinearities involving gradient terms. J. Optim. Theory Appl. 110 (2001) 245-264.

[5] J.M. Coron, Control and Nonlinearity. American Mathematical Society, Providence, RI (2007).

[6] L. De Teresa, Approximate controllability of a semilinear heat equation in $\mathbb{R}^{N}$. SIAM J. Control Optim. 36 (1998) $2128-2147$.

[7] L. De Teresa and E. Zuazua, Approximate controllability of a semilinear heat equation in unbounded domains. Nonlinear Anal. 37 (1999) 1059-1090. 
[8] Y. Duan, L. Wang and C. Zhang, Observability inequalities for the heat equation with bounded potentials on the whole space. SIAM J. Control Optim. 58 (2020) 1939-1960.

[9] M. Egidi and I. Veselićm Sharp geometric condition for null-controllability of the heat equation on $\mathbb{R}^{d}$ and consistent estimates on the control cost. Arch. Math. (Basel) 111 (2018) 85-99.

[10] M. Egidi, I. Nakić, A. Seelmann, M. Täufer, M. Tautenhahn and I. Veselić, Null-controllability and control cost estimates for the heat equation on unbounded and large bounded domains. Edited by J. Kerner, H. Laasri, D. Mugnolo, in Vol. 277 of Control Theory of Infinite-Dimensional Systems. Operator Theory: Advances and Applications. Birkhäuser, Cham (2020).

[11] C. Fabre, J. Puel and E. Zuazua, Approximate controllability of the semilinear heat equation. Proc. Roy. Soc. Edinburgh Sect. A 125 (1995) 31-61.

[12] E. Fernández-Cara and E. Zuazua, Null and approximate controllability for weakly blowing up semilinear heat equations. Ann. Inst. H. Poincaré Anal. Non Linéaire 17 (2000) 583-616.

[13] A.V. Fursikov and O.Y. Imanuvilov, Controllability of Evolution Equations. Seoul National University, Research Institute of Mathematics, Global Analysis Research Center, Seoul (1996).

[14] M. Gonzalez-Burgos and L. De Teresa, Some results on controllability for linear and nonlinear heat equations in unbounded domains. Adv. Differ. Equ. 12 (2007) 1201-1240.

[15] K. Le Balc'h, Global null-controllability and nonnegative-controllability of slightly superlinear heat equations. J. Math. Pures Appl. 135 (2020) 103-139.

[16] J. Le Rousseau and I. Moyano, Null-controllability of the Kolmogorov equation in the whole phase space. J. Differ. Equ. 260 (2016) 3193-3233.

[17] I. Nakić, M. Täufer, M. Tautenhahn and I. Veselić, Sharp estimates and homogenization of the control cost of the heat equation on large domains. ESAIM: COCV 26 (2020) 54.

[18] K.D. Phung, Carleman commutator approach in logarithmic convexity for parabolic equations. Math. Control Relat. Fields 8 (2018) 899-933.

[19] K.D. Phung and G. Wang, Quantitative unique continuation for the semilinear heat equation in a convex domain. J. Funct. Anal. 259 (2010) 1230-1247.

[20] K.D. Phung and G. Wang, An observability estimate for parabolic equations from a measurable set in time and its applications. J. Eur. Math. Soc. 15 (2013) 681-703.

[21] K.D. Phung, L. Wang and C. Zhang, Bang-bang property for time optimal control of semilinear heat equation. Ann. Inst. Henri Poincaré Anal. Non Linéaire 31 (2014) 477-499.

[22] J.C. Robinson, J.L. Rodrigo and W. Sadowski, The Three-Dimensional Navier-Stokes Equations. Classical Theory. Cambridge Studies in Advanced Mathematics, 157. Cambridge University Press, Cambridge (2016).

[23] A. Seelmann and I. Veselić, Exhaustion approximation for the control problem of the heat or Schrödinger semigroup on unbounded domains. Arch. Math. (Basel) 115 (2020) 195-213.

[24] G. Wang, L. Wang, Y. Xu and Y. Zhang, Time Optimal Control of Evolution Equations. Progress in Nonlinear Differential Equations and Their Applications, 92. Subseries in Control. Birkhäuser, Cham (2018).

[25] G. Wang, M. Wang, C. Zhang and Y. Zhang, Observable set, observability, interpolation inequality and spectral inequality for the heat equation in $\mathbb{R}^{N}$. J. Math. Pures Appl. 126 (2019) 144-194.

[26] G. Wang and C. Zhang, Observability inequalities from measurable sets for some abstract evolution equations. SIAM J. Control Optim. 55 (2017) 1862-1886.

[27] C. Zhang, Quantitative unique continuation for the heat equation with Coulomb potentials. Math. Control Relat. Fields 8 (2018) 1097-1116.

[28] X. Zhang and E. Zuazua, On the optimality of the observability inequalities for Kirchhoff plate systems with potentials in unbounded domains. Hyperbolic problems: theory, numerics, applications. Springer, Berlin (2008) 233-243.

\section{Subscribe to Open (S20) A fair and sustainable open access model}

This journal is currently published in open access under a Subscribe-to-Open model (S2O). S2O is a transformative model that aims to move subscription journals to open access. Open access is the free, immediate, online availability of research articles combined with the rights to use these articles fully in the digital environment. We are thankful to our subscribers and sponsors for making it possible to publish this journal in open access, free of charge for authors.

\section{Please help to maintain this journal in open access!}

Check that your library subscribes to the journal, or make a personal donation to the $\mathrm{S} 2 \mathrm{O}$ programme, by contacting subscribers@edpsciences.org

More information, including a list of sponsors and a financial transparency report, available at: https://www.edpsciences.org/en/maths-s2o-programme 